\title{
Uniqueness and the Global Markov Property for Euclidean Fields. The Case of Trigonometric Interactions
}

\author{
Sergio Albeverio* and Raphael Høegh-Krohn \\ Centre de Physique Théorique, CNRS, and Université d'Aix-Marseille II, UER de Luminy, \\ F-Marseille, France \\ Fakultat für Mathematik, Universität Bielefeld, D-4800 Bielefeld, Federal Republic of Germany \\ Matematisk Institutt, Universitetet i Oslo, Oslo, Norway
}

\begin{abstract}
We prove the global Markov property for the Euclidean measure given by weak trigonometric interactions. To obtain this result we first prove a uniqueness theorem concerning the set of regular Gibbs measures corresponding to a given interaction.
\end{abstract}

\section{Introduction}

The interest in extending the theory of Markov processes to the case where the time parameter is more than one dimensional, the so called Markov fields, arises in several domains, in particular in connection with problems of statistical mechanics, information theory and quantum field theory. The two main problems in the theory of Markov processes, the existence and uniqueness of the limit distribution and the question of the global Markov property are also main problems in the theory of Markov fields. For instance the question of the existence of a limit distribution corresponds in statistical mechanics and quantum field theory to the question of the existence of a Gibbs measure, while the uniqueness corresponds to the question whether this Gibbs measure defines a pure physical phase, non uniqueness indicating phase transitions.

The global Markov property on the other hand implies in statistical mechanics the existence of the transfer operator, and in quantum field theory it implies that the field theory is canonical and the zero time fields generate a maximal abelian subalgebra.

In this work we first prove that in the case of the Euclidean fields on $\mathbb{R}^{2}$ with weak trigonometric interaction one has uniqueness and also the global Markov property.

Concerning uniqueness corresponding results were proved in certain lattice and continuous models of statistical mechanics first by Dobrushin [13] (for further discussions see [14] and [15]).

* Postal address: Fakultät für Mathematik, Universität Bielefeld, D-4800 Bielefeld 1, Federal Republic of Germany 
Concerning the global Markov property this is proved for some lattice models by us in cooperation with Olsen [24] utilizing the ideas of the present paper.

The global Markov property for the Euclidean quantum field $\xi(x)$ in $\mathbb{R}^{2}$ is the following. Let $C$ be any piecewise $C^{1}$-curve such that $\mathbb{R}^{2}-C$ consists of two components $\Omega^{+}$and $\Omega^{-}$. Let $f_{+}$and $f_{-}$be any measurable functions of the field $\xi(x)$ which are measurable in $\Omega^{+}$and $\Omega^{-}$respectively. $\xi$ has the global Markov property iff

$$
E\left(f_{+} f_{-} \mid C\right)=E\left(f_{+} \mid C\right) E\left(f_{-} \mid C\right),
$$

where $E(f \mid C)$ is the conditional expectation of $f$ with respect to the $\sigma$-algebra generated by $C$ ("given all observations on $C$, observations within $\Omega^{+}$are conditionally independent of those within $\Omega^{-}$"). By taking $C$ from the family of lines orthogonal to a fixed direction we see that (1.1) implies that $\xi$ generates a Markov process in this direction.

Nelson observed [1] that (1.1) holds for the free Euclidean field as well as the nonhomogeneous random fields obtained by using multiplicative functionals of the free field. These are the so-called space-time cut-off quantum fields with polynomial [2], exponential [3] or trigonometric [4] interactions. The main interest in quantum field theory is naturally in the corresponding homogeneous (i.e. Euclidean invariant) random fields obtained as limits (the thermodynamical limit) of the above models. That these limits are Markov fields in the sense that (1.1) is satisfied whenever $C$ is a bounded curve such that $\mathbb{R}^{2}-C$ has two components, was first observed by Newman [8], who called this property the "local Markov property" (see also e.g. [9], [25]). For other properties weaker than the global Markov property see [6] and [10]-[12].

Let us now shortly describe the methods by which we prove our results, starting with the one about the uniqueness of the Gibbs measures. The measures we consider are of the type $\left(E_{0}(\cdot) \equiv \int \cdot d \mu_{0}\right)$

$$
d \mu_{\Lambda}=E_{0}\left(e^{-U_{\Lambda}}\right)^{-1} e^{-U_{\Lambda}} d \mu_{0} .
$$

where $d \mu_{0}$ is the free Euclidean field measure on $S^{\prime}\left(\mathbb{R}^{2}\right)$ [mean zero and covariance $\left.\left(-\Delta+m^{2}\right)^{-1}\right]$, and $U_{A}$ is a local space-time cut off interaction, so that $\Lambda \rightarrow U_{A}$ is an additive map from the bounded Borel subsets of $\mathbb{R}^{2}$ into $L^{1}\left(d u_{0}\right)$ with

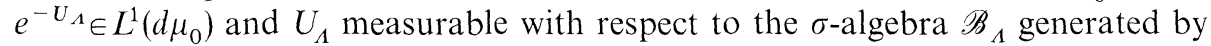
the fields with support within $\Lambda$. A Gibbs measure associated with $U_{\Lambda}$ is any measure which is locally absolutely continuous with respect to $\mu_{0}$ and such that the associated conditional expectation of functions measurable within $\Lambda$ with respect to the $\sigma$-algebra $\mathscr{B}_{\hat{c} A}$, coincides with those computed with $\mu_{A}$ instead of $\mu$, shortly

$$
E_{\mu}(f \mid \partial \Lambda)=E_{\mu_{\Lambda}}(f \mid \partial \Lambda) .
$$

Such measures are also said to satisfy the DLR-equations, see $[13,20,16]$. In particular any weak limit point of measures of the form $\mu_{A}$ is a Gibbs measure and one sees that any Gibbs measure has the local Markov property. A Gibbs measure is defined to be pure if there is no other Gibbs measure absolutely continuous with respect to it. We show, using the local Markov property and the reverse martingale 
theorem, that purity of $\mu$ is equivalent with $E_{\mu}(f \mid \partial \Lambda) \rightarrow E(f) \mu$-almost surely as $\Lambda \uparrow \mathbb{R}^{2}$. These concepts and the results are given in Sect. 3 . The actual proof of the convergence

$$
E_{\mu}(f \mid \partial \Lambda) \rightarrow E(f)
$$

uses the following basic idea. By (1.3) and the definition of $\mu_{A}$ we have

$$
E_{\mu}(f \mid \partial \Lambda)=E_{\mu_{\Lambda}}(f \mid \partial \Lambda)=\frac{E_{0}\left(f e^{-U_{\Lambda}} \mid \partial \Lambda\right)}{E_{0}\left(e^{-U_{\Lambda}}\right)}
$$

On the other hand one obtains a more explicit expression for the right hand side in the following way.

Let $C \equiv \partial \Lambda$ and let $P_{C}(x, z)$ be the Poisson kernel associated with the Dirichlet problem

$$
\left(-\Delta+m^{2}\right) \psi_{g}^{C}(x)=0, \quad x \in \mathbb{R}^{2}-C
$$

and the boundary condition

$$
\psi_{g}^{C}(x)=g(x), \quad x \in C .
$$

Then for $g \in C_{0}\left(\mathbb{R}^{2}\right)$ we have that $\psi_{g}^{C}(x)=\int_{C} P_{C}(x, z) g(z) d z$. On the other hand we prove that, for $x \in \mathbb{R}^{2}-C, \xi \rightarrow \psi_{\xi}^{C}(x)$ is a $\mu_{0}$-measurable linear function and in fact with the help of the Gaussian random field $x \rightarrow \psi_{\xi}^{C}(x)$, where $\xi$ is the free Euclidean field, it is possible to give an explicit formula for $E_{0}(\cdot \mid C)$ by

$$
E_{0}(f \mid C)(\eta)=E_{\mu_{0}}\left(f\left(\xi+\psi_{\eta}^{c}\right)\right)
$$

for $\mu_{0}$-almost all $\eta$, where $\mu_{0}^{c}$ is the free (Gaussian) measure with Dirichlet boundary conditions on $C$. That is $\mu_{0}^{C}$ is the Gaussian measure of mean zero and covariance $\left(-\Lambda_{C}+m^{2}\right)^{-1}$, where $\Delta_{C}$ is the Laplacian with Dirichlet boundary conditions on C.

Introducing then (1.5) into (1.4) we get

$$
E_{\mu}(f \mid \partial \Lambda)(\eta)=E_{\mu_{\eta}^{i \Lambda}}\left(f^{\eta}\right)
$$

with

$$
d \mu_{\eta}^{i A}(\xi) \equiv E_{\mu_{0}^{C}}\left(e^{\left.-U_{\Lambda}^{n}\right)^{-1}} e^{-U_{\Lambda}^{n}(\xi)} d \mu_{0}^{C}(\xi),\right.
$$

where for any function $g(\xi)$ on $S^{\prime}\left(\mathbb{R}^{2}\right)$ we use the notation $g^{\eta}(\xi) \equiv g\left(\xi+\psi_{\eta}^{\hat{o} \Lambda}\right)$. For the family of functions $f(\xi) \equiv e^{i\langle\xi, \varphi\rangle}, \varphi \in S\left(\mathbb{R}^{2}\right)$, we have then

$$
E_{\mu}\left(e^{i\langle\xi, \varphi\rangle} \mid \partial \Lambda\right)(\eta)=e^{i\left\langle\psi_{\eta}^{\Lambda \Lambda}, \varphi\right\rangle} \int e^{i\langle\xi, \varphi\rangle} d \mu_{\eta}^{\hat{i} \Lambda}(\xi) .
$$

Thus the proof of $E_{\mu}(f \mid \partial \Lambda) \rightarrow E(f)$ as $\Lambda \uparrow \mathbb{R}^{2}$ amounts to the proof of

A) $\left\langle\psi_{\eta}^{\hat{i} \Lambda}, \varphi\right\rangle \rightarrow 0$ for $\mu$-a.e. $\eta$,

B) $\mu_{\eta}^{i A} \rightarrow \mu$, weakly as $\Lambda \uparrow \mathbb{R}^{2}$, for $\mu$-a.e. $\eta$.

The proof of $\mathrm{A}$ ) is obtained under the condition that $\mu$ be a Gibbs measure satisfying a suitable regularity condition (second moments bounded by those of some free Euclidean field: this condition is verified in all applications), by using $L^{2}(d \mu)$-estimates on the field $\psi_{\eta}^{C}(x)$. This is done in Sect. 2, where we prove in fact 
that, for $\mu$-a.e. $\eta, \psi_{\eta}^{C}(x)$ goes to zero uniformly in $x$ exponentially fast as the distance from $x$ to $C$ tends to infinity.

The proof of $\mathrm{B}$ ) is obtained for the choice of interaction

$$
U_{\Lambda}(\xi)=\lambda \int_{\Lambda}: \cos (\alpha \xi(x)+\theta): d x
$$

with $\lambda, \alpha, \theta$ real, $\alpha^{2}<2 \pi, 0 \leqq \theta<2 \pi$ and $\lambda$ sufficiently small (depending on $\alpha$ and $m$ ). The method of the proof is an adaption of the cluster expansion method first introduced by Glimm, Jaffe and Spencer [2] for the polynomial interaction and developed by Fröhlich and Seiler [4] for the trigonometric interaction (1.9). We need, in order to prove B), to extend the method by Fröhlich and Seiler to cover the case of the interaction $U_{A}^{\eta}(\xi)$ for $\mu$-almost all $\eta$, rather then of the $U_{A}(\xi)$ interaction (1.9), where we recall that $U_{\Lambda}^{\eta}(\xi) \equiv U_{\Lambda}\left(\xi+\psi_{\eta}^{C}\right)$. The technical estimates required for this extension are all given in Sect. 4 , which is the most technical section of this paper. For the reader's convenience we have tried to keep the notations in this section and the proofs as close as possible to the corresponding one in [4].

In Sect. 5 we combine in the described way, the results of the Sect. 2, Sect. 3 and Sect. 4 to yield the uniqueness result for a Gibbs measure associated with the interactions (1.9).

In Sect. 6 we prove the global Markov property for the trigonometric interactions. The ideas of this proof are the following. Let $C$ be a fixed piecewise $C^{1}$-curve and $\mu$ a Gibbs measure for the trigonometric interaction. We assume that $\mathbb{R}^{2}-C$ consists of two components $\Omega^{+}$and $\Omega^{-}$. Let $\Lambda$ be a bounded subset and $f_{ \pm}$ be measurable in $\Lambda \cap \Omega^{ \pm}$respectively. By the definition of a Gibbs measure we then have

$$
E_{\mu}\left(f_{+} f_{-} \mid C \cup \partial \Lambda\right)=E_{\mu_{\Lambda}}\left(f_{+} f_{-} \mid C \cup \partial \Lambda\right)
$$

and by the global Markov property of $\mu_{\Lambda}$ we have that (1.10) is equal to

$$
E_{\mu_{\Lambda}}\left(f_{+} \mid C \cup \partial \Lambda\right) E_{\mu_{\Lambda}}\left(f_{-} \mid C \cup \partial \Lambda\right)=E_{\mu}\left(f_{+} \mid C \cup \partial \Lambda\right) E_{\mu}\left(f_{-} \mid C \cup \partial \Lambda\right) .
$$

If now $\mu_{\eta}^{c}$ is the measure $\mu$ conditioned by $\xi=\eta$ on $C$ then (1.11) is equal to

$$
E_{\mu_{\eta}^{c}}\left(f_{+} \mid \partial \Lambda\right) E_{\mu_{\eta}^{c}}\left(f_{-} \mid \partial \Lambda\right)
$$

Now $\mu_{\eta}^{C}$ is in fact a Gibbs measure corresponding to the interaction $U_{A}\left(\xi+\psi_{\eta}^{C}\right)$. Hence we know that

$$
E_{\mu_{\eta}^{C}}^{C}\left(f_{ \pm} \mid \partial \Lambda\right) \rightarrow E_{\mu_{\eta}^{C}}\left(f_{ \pm}\right)=E_{\mu}\left(f_{ \pm} \mid C\right)(\eta)
$$

if and only if $\mu_{\eta}^{C}$ is a pure Gibbs measure for the interaction $U_{A}\left(\xi+\psi_{\eta}^{C}\right)$.

We now proceed to prove that, for $\mu$-almost all $\eta, \mu_{\eta}^{C}$ is pure by extending the estimates and techniques for establishing uniqueness developed in Sect. 5 to cover also this situation. Hence we have the convergence (1.13) which by the equality of (1.10) and (1.12) proves that

$$
E_{\mu}\left(f_{+} f_{-} \mid C\right)=E_{\mu}\left(f_{+} \mid C\right) E_{\mu}\left(f_{-} \mid C\right)
$$

i.e. the global Markov property. 
Let us now add some remarks on our results and relations to other works. Concerning the uniqueness we show that there is a unique Gibbs measure within the class of regular random fields (i.e. fields with two point functions dominated by some free two point functions). We also show for the same trigonometric interaction that with boundary condition $\xi=\eta$ on $\partial \Lambda$, where $\eta$ is any fixed element in $S^{\prime}\left(\mathbb{R}^{2}\right)$ such that $\psi_{\eta}^{\partial \Lambda}(x)$ exists for $x \in \mathbb{R}^{2}-\partial \Lambda$ and converges locally uniformly to zero as $\Lambda \uparrow \mathbb{R}^{2}$, the corresponding measures converge weakly to this Gibbs measure, which is thus independent of boundary conditions. Results on independence of some particular boundary conditions were obtained for the polynomial interactions in [26] [28].

Concerning the global Markov property we remark that consequences of it have been discussed in several papers, based on postulates i.e. assuming it to hold together with some general properties, see e.g. $[7,16,6 \mathrm{a}]$ ([5] contains further references). In the present case all assumptions hold, hence all conclusions are now proven for the trigonometric interactions. In particular we have that the corresponding quantum fields are canonical ones and the time zero fields generate the physical Hilbert space, i.e. the weakly closed algebra generated by the time zero fields is a maximal abelian algebra ("cyclicity of the time zero fields"). From the global Markov property it follows also, as pointed out by us in previous work ([5, $17,19]$, to which we refer for more details) that the physical Hamiltonian is a second order elliptic variational operator, i.e. an infinite dimensional analog of the Schrödinger operator of quantum mechanics. In fact the whole "Schrödinger representation" of this theory holds, which completely justifies the ideas of the canonical formalism [18] for this field theory. Let us finally remark that the essential ideas for proving the global Markov property have been applied by us, in collaboration with Olsen, to the case of the lattice model of statistical mechanics, yielding the first proof of the existence of a transfer matrix in this case [24].

\section{Regular Random Fields on $\mathbb{R}^{2}$}

Let $\Delta=\frac{\partial^{2}}{\partial x_{1}^{2}}+\frac{\partial^{2}}{\partial x_{2}^{2}}$ be the self-adjoint Laplacian on $L_{2}\left(\mathbb{R}^{2}\right)$ and let $G_{m}(x-y)$ be the kernel of the operator $\left(-\Delta+m^{2}\right)^{-1}$, where $m>0$. Let $\varrho$ be a positive bounded Borel measure of bounded support in $\mathbb{R}^{2}$ and set

$$
E_{m}(\varrho)=\int G_{m}(x-y) d \varrho(x) d \varrho(y) .
$$

Since $G_{m}(x)$ is a positive continuous function for $x \neq 0$ and $G_{m}(x) \rightarrow 0$ as $|x| \rightarrow \infty$, $G_{m}(x-y)$ is Borel-measurable and therefore $E_{m}(\varrho)$ is well defined with values in the extended positive half line. We say that $\varrho$ has bounded energy if $E_{m}(\varrho)<\infty$. Since $\varrho$ has bounded support, we see that if $E_{m_{0}}(\varrho)<\infty$ for some positive number $m_{0}$ than $E_{m}(\varrho)<\infty$ for all $m>0$. We denote the set of positive bounded Borel measures on $\mathbb{R}^{2}$ with bounded support and bounded energy by $M_{b}^{+}$and we denote its linear span by $M_{b}$. We see that $M_{b}^{+}$and hence $M_{b}$ do not depend on the positive number $m$.

Let $S^{\prime}\left(\mathbb{R}^{2}\right)$ be the space of tempered distributions on $\mathbb{R}^{2}$ and let $S\left(\mathbb{R}^{2}\right)$ be its dual. Let $\mu$ be a Borel probability measure on $S^{\prime}\left(\mathbb{R}^{2}\right)$, where $S^{\prime}\left(\mathbb{R}^{2}\right)$ is given the 
Borel $\sigma$-algebra $B$ generated by the open sets in $S^{\prime}\left(\mathbb{R}^{2}\right)$. The corresponding $S^{\prime}\left(\mathbb{R}^{2}\right)$ valued random variable $\xi(x)$ is called a tempered random field on $\mathbb{R}^{2}$. We say that a tempered random field $\xi(x)$ is regular if there exist a number $m>0$ and a constant $c$ such that for any $\varphi \in S\left(\mathbb{R}^{2}\right)$ we have

$$
\int|\langle\varphi, \xi\rangle|^{2} d \mu(\xi) \leqq c E_{m}(\varphi),
$$

where $\langle\varphi, \xi\rangle$ is the dualization between $S\left(\mathbb{R}^{2}\right)$ and $S^{\prime}\left(\mathbb{R}^{2}\right)$. Note that if $m \geqq m_{0}>0$ then $E_{m}(\varphi) \leqq E_{m_{0}}(\varphi) \leqq\left(\frac{m}{m_{0}}\right)^{2} E_{m}(\varphi)$, so that the condition (2.2) is independent of $m$.

Let now $\xi(x)$ be a fixed regular random field on $\mathbb{R}^{2}$ and let $\mu$ be the corresponding probability measure on $S^{\prime}\left(\mathbb{R}^{2}\right)$. It follows then from (2.2) that $\xi \rightarrow\langle\varrho, \xi\rangle$ for $\varrho \in M_{b}\left(\mathbb{R}^{2}\right)$ is in $L_{2}(\mu) \equiv L_{2}\left(S^{\prime}\left(\mathbb{R}^{2}\right), \mu\right)$. Hence for any $\varrho \in M_{b}\left(\mathbb{R}^{2}\right)$ we may take $\langle\varrho, \xi\rangle$ to be Borel measurable by modifying it at some set of $\mu$-measure zero. For any Borel set $\Lambda \subset \mathbb{R}^{2}$ we define $M_{b}(\Lambda)$ to be the subspace of $M_{b}\left(\mathbb{R}^{2}\right)$ consisting of measures $\varrho \in M_{b}\left(\mathbb{R}^{2}\right)$ with supp $\varrho \subset \Lambda$. Moreover let $B_{A}$ be the $\sigma$ algebra of subsets of $S^{\prime}\left(\mathbb{R}^{2}\right)$ generated by the linear functions $\xi \rightarrow\langle\varrho, \xi\rangle$ with $\varrho \in M_{b}(\Lambda)$. The map $\Lambda \rightarrow B_{A}$ is then a monotone map from the $\sigma$-algebra of Borel subsets of $\mathbb{R}^{2}$ into the ordered set of $\sigma$-subalgebras of $B=B_{\mathbb{R}^{2}}$. Therefore if $E(\cdot \mid \Lambda)$ is the conditional expectation with respect to the $\sigma$-algebra $B_{A}$, then $\Lambda \rightarrow E_{A}$ is a monotone map from Borel subsets of $\mathbb{R}^{2}$ into the ordered set of orthogonal projections on $L_{2}(\mu)$. Hence if $\Lambda_{n}$ is a monotone (increasing or decreasing) sequence of Borel subsets of $\mathbb{R}^{2}$, then $E\left(\cdot \mid \Lambda_{n}\right)$ is a monotone hence strongly convergent sequence of orthogonal projections in $L_{2}(\mu)$. Since $A_{n}$ is monotone we have in fact that $E\left(f \mid \Lambda_{n}\right)$ for $f \in L_{1}(\mu)$ is a uniformly integrable martingale and therefore by the martingale convergence theorem $E\left(f \mid \Lambda_{n}\right)$ converges almost surely and also strongly in $L_{1}(\mu)$. For the martingale convergence theorem see e.g. [29]. Hence we have the following Lemma.

Lemma 2.1. Let $\Lambda_{n}$ be a monotone (increasing or decreasing) sequence of Borel subsets of $\mathbb{R}^{2}$. Then for any $f \in L_{1}(\mu)$ we have that $E\left(f \mid \Lambda_{n}\right)$ converges $\mu$-almost surely and also strongly in $L_{1}(\mu)$.

Let now $C$ be a piecewise $C^{1}$-curve in $\mathbb{R}^{2}$, and let $g \in C_{0}\left(\mathbb{R}^{2}\right)$, where $C_{0}\left(\mathbb{R}^{2}\right)$ denotes the continuous functions with compact support on $\mathbb{R}^{2}$. Let $\psi_{g}^{C}(x)$ be the unique solution of

$$
\left(-\Delta+m^{2}\right) \psi_{g}^{C}(x)=0 \text { for } \quad x \in \mathbb{R}^{2}-C,
$$

with

$$
\psi_{g}^{C}(z)=g(z) \text { for } z \in C,
$$

where $m$ is the positive number occurring in (2.2).

It is well known, see for instance [30], that $\psi_{q}^{C}(x)$ exists and, for fixed $x \in \mathbb{R}^{2}-C$, that $g \rightarrow \psi_{g}^{C}(x)$ is a positive continuous linear function on $C_{0}\left(\mathbb{R}^{2}\right)$. The correspond- 
ing measure is absolutely continuous with respect to the natural measure $d z$ on $C$ i.e.

$$
\psi_{g}^{C}(x)=\int_{C} P_{C}(x, z) g(z) d z,
$$

where the Poisson kernel $P_{C}(x, z)$ is a measurable function on $\mathbb{R}^{2} \times C$. For $x \notin C$ we call $\psi_{\xi}^{C}(x)$ the linear function $\xi \rightarrow \psi_{\xi}^{C}(x)$ of the random field given by (2.4) i.e.

$$
\psi_{\xi}^{C}(x)=\int_{C} P_{C}(x, z) \xi(z) d z .
$$

We then find

$$
\int_{S^{\prime}\left(\mathbb{R}^{2}\right)} \psi_{\xi}^{C}(x) \psi_{\xi}^{C}(y) d \mu(\xi)=\iint_{C} P_{C}\left(x, z_{1}\right) S_{2}\left(z_{1}, z_{2}\right) P_{C}\left(y, z_{2}\right) d z_{1} d z_{2},
$$

where

$$
S_{2}(x, y)=\int \xi(x) \xi(y) d \mu(\xi)
$$

is the second moment of $\mu$. From (2.2) we have that, for $f \in S\left(\mathbb{R}^{2}\right)$,

$$
\iint S_{2}(x, y) f(x) f(y) d x d y \leqq c E_{m}(f) .
$$

Therefore if $\psi_{\xi}^{C}(\varphi)=\int \psi_{\xi}^{C}(x) \varphi(x) d x$ for $\varphi \in S\left(\mathbb{R}^{2}\right)$ and if $\operatorname{supp} \varphi \subset \mathbb{R}^{2}-C$, then by (2.5) and (2.7) we have that

$$
\int_{S^{\prime}\left(\mathbb{R}^{2}\right)}\left|\psi_{\xi}^{C}(\varphi)\right|^{2} d \mu(\xi) \leqq c \iint K_{C}(x, y) \varphi(x) \varphi(y) d x d y
$$

with

$$
K_{C}(x, y)=\int_{C} \int_{C} P_{C}\left(x, z_{1}\right) G_{m}\left(z_{1}-z_{2}\right) P_{C}\left(y, z_{2}\right) d z_{1} d z_{2}
$$

Since $G_{m}(x-z), z \in C$ is a solution of (2.3) with boundary condition $g(w)$ $=G_{m}(w-z)$, we get from (2.4) that for $x$ and $y$ in $\mathbb{R}^{2}-C$

$$
K_{C}(x, y)=\int_{C} G_{m}(x-z) P_{C}(y, z) d z .
$$

We have from (2.9) that $K_{C}(x, y)=K_{C}(y, x)$ and $\left(-\Delta_{x}+m^{2}\right) K_{C}(x, y)=0$ on $\mathbb{R}^{2}-C$ $-\{y\}$. Moreover from (2.10) and (2.4) we see that if $x \in \mathbb{R}^{2}-C$ then $K_{C}(x, z)=G_{m}(x-z)$ for $z \in C$. This however implies that

$$
K_{C}(x, y)=G_{m}(x-y)-G_{m}^{C}(x, y)
$$

where $G_{m}^{C}(x, y)$ is the kernel of the operator $\left(-\Delta_{C}+m^{2}\right)^{-1}, \Delta_{C}$ being the Laplacian with Dirichlet boundary conditions on the curve $C$. It is well known that $K_{C}(x, y)$ is a bounded continuous function of $x$ an $y$ in $\mathbb{R}^{2}-C$. This follows from the fact that $G_{m}(x-y)$ and $G_{m}^{C}(x, y)$ are both of the form $-\frac{1}{2 \pi} \ln |x-y|+f(x, y)$, where $f(x, y)$ is a bounded continuous function. Especially we get from (2.8) that if $x \notin C$ then

$$
E\left(\left|\psi_{\xi}^{C}(x)\right|^{2}\right) \leqq c K_{C}(x, x),
$$


where $E$ stands for the expectation with respect to the random field $\xi$. From the known behavior of $G_{m}(x-y)$ and $G_{m}^{C}(x, y)$ we have that there is a constant $b$ such that

$$
\left|K_{C}(x, x)\right| \leqq b|\ln d(x, C)| \cdot e^{-m d(x, C)}
$$

where $d(x, C)$ is the distance from $x$ to $C$. Therefore

$$
E\left(\left|\psi_{\xi}^{C}(x)\right|^{2}\right) \leqq a|\ln d(x, C)| \cdot e^{-m d(x, C)}
$$

where $a=b \cdot c$.

Let now $\Lambda$ be a compact subset of $\mathbb{R}^{2}-C$, such that $d(\Lambda, C) \geqq 1$. From (2.14) we then get

$$
E\left(\int_{\Lambda}\left|\psi_{\xi}^{C}(x)\right|^{2} d x\right) \leqq a^{\prime}|\Lambda| e^{-m^{\prime} d(\Lambda, C)}
$$

where $|\Lambda|$ is the volume of $A$ and $m^{\prime}<m$. Hence we have the following lemma.

Lemma 2.2. Let $\xi(x)$ be a regular random field on $\mathbb{R}^{2}$ so that

$$
E\left(|\langle\varrho, \xi\rangle|^{2}\right) \leqq c E_{m}(\varrho)
$$

for any $\varrho \in M_{b}$. Let $C$ be a piecewise $C^{1}$-curve in $\mathbb{R}^{2}$ and let $\psi_{g}^{C}(x)$ be the solution of the Dirichlet problem (2.3). Then, for any $x \in \mathbb{R}^{2}-\bar{C}, \xi \rightarrow \psi_{\xi}^{C}(x)$ is in $L_{2}(\mu)$, where $\mu$ is the probability measure on $S^{\prime}\left(\mathbb{R}^{2}\right)$ given by $\xi$, and there is a constant a such that

$$
E\left(\left|\psi_{\xi}^{C}(x)\right|^{2}\right) \leqq a|\ln (d(x, C))| e^{-m d(x, C)} .
$$

Moreover if $\Lambda$ is a compact subset of $\mathbb{R}^{2}-C$ such that $d(\Lambda, C) \geqq 1$ then

$$
E\left(\int_{\Lambda}\left|\psi_{\xi}^{C}(x)\right|^{2} d x\right) \leqq a^{\prime}|\Lambda| e^{-m^{\prime} d(\Lambda, C)}, m^{\prime}<m
$$

Let now $\psi$ satisfy the equation $\left(-\Delta+m^{2}\right) \psi=0$ for $|x|<R$. Let $\psi_{0}$ be the function obtained from $\psi$ by averaging with respect to rotations i.e.

$$
\psi_{0}(x)=\int_{\operatorname{SO}(2)} \psi(\sigma x) d \sigma .
$$

Since $-\Delta+m^{2}$ is invariant with respect to $\mathrm{SO}(2)$ we have that $\left(-\Delta+m^{2}\right) \psi_{0}=0$. By rotational invariance we have $\psi_{0}(x)=\varphi(|x|)$ and $\left(-\Delta+m^{2}\right) \psi_{0}=0$ gives us the equation

$$
\varphi^{\prime \prime}(r)+\frac{1}{r} \varphi^{\prime}(r)=m^{2} \varphi(r) .
$$

(2.17) has a regular singularity at $r=0$ and there is therefore a unique solution $\varphi_{0}(r)$ of (2.17) defined for $r \geqq 0$ such that $\varphi_{0}(0)=1$. But then we have that $\psi_{0}(x)=\psi_{0}(0) \cdot \varphi_{0}(|x|)$ i.e., by the definition of $\psi_{0}$,

$$
\frac{1}{2 \pi r} \int_{|x|=r} \psi_{0}(x) d x=\varphi_{0}(r) \cdot \psi_{0}(0) .
$$


Now we observe that $\varphi_{0}(r)>0$ for all $r \geqq 0$. (This is so because if we would like $\varphi_{0}(a)=0$ for $a>0$ then this would imply that $\psi_{0}(x)=\varphi_{0}(|x|)$ is a solution of $\left(-\Delta+m^{2}\right) \psi_{0}=0$ such that $\psi_{0}=0$ for $|x|=a$. By partial integration we then would get that

$$
\int_{|x| \leqq a}\left|\nabla \psi_{0}\right|^{2} d x=-m^{2} \int_{|x| \leqq a}\left|\psi_{0}\right|^{2} d x
$$

which is a contradiction.) Using now the translational invariance of $-\Delta+m^{2}$ we have the following lemma

Lemma 2.3. Let $\alpha_{m}(r)=\varphi_{0}(r)^{-1}$ where $\varphi_{0}(r)$ is the unique solution for $r \geqq 0$ of the equation $\varphi_{0}^{\prime \prime}(r)+\frac{1}{r} \varphi_{0}^{\prime}(r)=m^{2} \varphi_{0}(r)$ such that $\varphi_{0}(0)=1$. Then if $\psi$ is a solution of the equation $\left(-\Delta+m^{2}\right) \psi=0$ for $|y-x|<R$ then

$$
\psi(x)=\frac{\alpha_{m}(r)}{2 \pi r} \int_{|x-y|=r} \psi(y) d y
$$

for $r \leqq R$.

It follows from the fact that $\varphi_{0}(r)$ is a solution of

$$
\varphi_{0}^{\prime \prime}(r)+\frac{1}{r} \varphi_{0}^{\prime}(r)=m^{2} \varphi_{0}(r)
$$

which remains bounded at $r=0$ that $\varphi_{0}^{\prime}(0)=0$. From $(2.20)$ we then get $\varphi_{0}^{\prime \prime}(0)=m^{2}$ hence $\varphi_{0}^{\prime}(r)>0$ for $r \in(0, \varepsilon)$ for some $\varepsilon>0$. Now since $\varphi_{0}(r)>0$ for all $r \geqq 0$ we see that at any point $r_{0}$ where $\varphi_{0}^{\prime}\left(r_{0}\right)=0$ one has $\varphi_{0}^{\prime \prime}\left(r_{0}\right)>0$ so that the function $\varphi_{0}(r)$ has no local maximum and is therefore monotone increasing i.e. $\varphi_{0}^{\prime}(r) \geqq 0$ for all $r \geqq 0$. Therefore $\alpha_{m}(r)$ is monotone decreasing and thus from Lemma 2.3 we get, with $\varrho \leqq r$ :

$$
|\psi(x)|^{2} \leqq \frac{\alpha_{m}(r)^{2}}{2 \pi r} \int_{|x-y|=r}|\psi(y)|^{2} d y \leqq \frac{\alpha_{m}(\varrho)^{2}}{2 \pi \varrho} \int_{|x-y|=r}|\psi(y)|^{2} d y .
$$

Now since $\int_{\varrho \leqq|x| \leqq \varrho+1} d x=\pi(\varrho+1)^{2}-\pi \varrho^{2}=2 \pi\left(\varrho+\frac{1}{2}\right)>2 \pi \varrho$ we get by integrating the right hand side with respect to $r$, between $\varrho$ and $\varrho+1$ :

$$
|\psi(x)|^{2} \leqq\left(\frac{\alpha_{m}(\varrho)}{2 \pi \varrho}\right)^{2} \int_{\varrho \leqq|x-y| \leqq \varrho+1}|\psi(y)|^{2} d y .
$$

From this we have the following lemma.

Lemma 2.4. Let $\alpha_{m}(r)$ be as in Lemma 2.3 and set

$$
\beta_{m}(r)=\left(\alpha_{m}(r) / 2 \pi r\right)^{2} .
$$

Then if $\psi(x)$ is a solution of the equation $\left(-\Delta+m^{2}\right) \psi=0$ in some open set $\Lambda \subset \mathbb{R}^{2}$ then for any $x \in \Lambda$ such that $d(x, \partial \Lambda)>1$ we have

$$
|\psi(x)|^{2} \leqq \beta_{m}(d(x, \partial \Lambda)-1) \int_{\Lambda}|\psi(y)|^{2} d y .
$$


Moreover $\beta_{m}(r)$ is a monotone decreasing function of $r$ such that

$$
\beta_{m}(r) \sim \frac{1}{(2 \pi r)^{2}} e^{-2 m r}
$$

as $r \rightarrow \infty$.

Let now $\xi(x)$ be a regular random field on $\mathbb{R}^{2}$, and let $C_{n}$ be a sequence of piecewise $C^{1}$-curves in $\mathbb{R}^{2}$ which tends to infinity in the sense that

$$
d\left(0, C_{n}\right) \rightarrow \infty .
$$

Then $d\left(\Lambda, C_{n}\right) \rightarrow \infty$ for any bounded $\Lambda \subset \mathbb{R}^{2}$. Let $\Lambda_{n}$ be a sequence of bounded subsets of $\mathbb{R}^{2}$ such that $\Lambda_{n}$ is contained in the interior of $C_{n}$. From Lemma 2.2 we have for any $\alpha<m$ that

$$
e^{\alpha d\left(\Lambda_{n}, C_{n}\right)} E\left(\int_{\Lambda_{n}}\left|\psi_{\xi}^{C_{n}}(x)\right|^{2} d x\right) \rightarrow 0
$$

as $n \rightarrow \infty$ if $d\left(\Lambda_{n}, C_{n}\right) \rightarrow \infty$. Thus the sequence of functions

$$
\xi \rightarrow e^{\alpha / 2 d\left(A_{n}, C_{n}\right)}\left(\int_{\Lambda_{n}}\left|\psi_{\xi}^{C_{n}}(x)\right|^{2} d x\right)^{1 / 2}
$$

converges to zero in $L_{2}(\mu)$ and therefore there is a subsequence that converges to zero $\mu$-almost everywhere. Let $n^{\prime}$ be this subsequence, then

$$
e^{\alpha d\left(\Lambda_{n^{\prime}}, C_{n^{\prime}}\right)} \int_{\Lambda_{n^{\prime}}}\left|\psi_{\xi}^{C_{n^{\prime}}}(x)\right|^{2} d x \rightarrow 0
$$

as $n^{\prime} \rightarrow \infty, \mu$-almost everywhere. Let $\tilde{\Lambda}_{n} \supset \Lambda_{n}$ such that $d\left(\Lambda_{n}, \partial \tilde{\Lambda}_{n}\right) \geqq 2$ and $\tilde{\Lambda}_{n}$ is contained in the interior of $C_{n}$.

By Lemma 2.4 we then have for $x \in \Lambda_{n}$ that

$$
\left|\psi_{\xi}^{C_{n}}(x)\right|^{2} \leqq \beta_{m}(1) \int_{\widetilde{X}_{n}}\left|\psi_{\xi}^{C_{n}}(y)\right|^{2} d y .
$$

We may obviously take $\tilde{\Lambda}_{n}$ so that $\frac{d\left(\tilde{\Lambda}_{n}, C_{n}\right)}{d\left(\Lambda_{n}, C_{n}\right)} \rightarrow 1$ and from (2.25) and (2.26) we then have that

$$
e^{\alpha / 2 d\left(\Lambda_{n^{\prime}}, C_{n^{\prime}}\right)} \sup _{x \in A_{n^{\prime}}}\left|\psi_{\xi}^{C_{n^{\prime}}}(x)\right| \rightarrow 0
$$

for $\mu$-almost all $\xi$.

We have proved the following theorem.

Theorem 2.1. Let $\xi(x)$ be a regular random field on $\mathbb{R}^{2}$, so that there is a constant $c$ such that for all $\varphi \in S\left(\mathbb{R}^{2}\right)$

$$
E\left(|\langle\varphi, \xi\rangle|^{2}\right) \leqq c E_{m}(\varphi) \equiv c \iint G_{m}(x-y) \varphi(x) \varphi(y) d x d y .
$$

If $C$ is a piecewise $C^{1}$-curve let $\psi_{g}^{C}(x)$ be the solution of $\left(-\Delta+m^{2}\right)=0$ in $\mathbb{R}^{2}-C$ with the boundary condition $\psi_{g}^{C}(z)=g(z)$ for $z \in C$. Then for $x \notin C, g \rightarrow \psi_{g}^{C}(x)$ is a linear 
functional on $C_{0}\left(\mathbb{R}^{2}\right)$ which extends to a $\mu$-measurable linear function $\xi \rightarrow \psi_{\xi}^{C}(x)$ which is in $L_{2}(\mu)$, where $\mu$ is the probability measure on $S^{\prime}\left(\mathbb{R}^{2}\right)$ given by the regular random field $\xi(x)$.

Moreover if $C_{n}$ is any sequence of piecewise $C^{1}$-curves in $\mathbb{R}^{2}$ which tends to infinity in the sense that $d\left(0, C_{n}\right) \rightarrow \infty$, and $\Lambda_{n}$ any sequence of bounded sets in $\mathbb{R}^{2}$ such that $\Lambda_{n}$ is contained in $\mathbb{R}^{2}-C_{n}$ and $d\left(\Lambda_{n}, C_{n}\right) \rightarrow \infty$ as $n \rightarrow \infty$, then for any $\alpha<m$ there is a subsequence $n^{\prime}$ such that

$$
e^{\alpha / 2 d\left(\Lambda_{n^{\prime}}, C_{n^{\prime}}\right)} \sup _{x \in \Lambda_{n^{\prime}}}\left|\psi_{\xi}^{C_{n^{\prime}}}(x)\right| \rightarrow 0
$$

for almost all $\xi$.

Let us now consider two piecewise $C^{1}$-curves $C_{0}$ and $C$ such that $C_{0} \cap C=\emptyset$. Then for $g \in C\left(\mathbb{R}^{2}\right)$

$$
\psi_{g}^{C, C_{0}}(x)=\psi_{g}^{C \cup C_{0}}(x)-\psi_{g}^{C_{0}}(x)
$$

is a solution of the equation

$$
\left(-\Delta+m^{2}\right) \psi=0
$$

in $\mathbb{R}^{2}-C \cup C_{0}$ such that

$$
\psi_{g}^{C, C_{0}}(z)=0 \quad \text { for } \quad z \in C_{0}, \psi_{g}^{C, C_{0}}(z)=g(z)-\psi_{g}^{C_{0}}(z) \text { for } \quad z \in C .
$$

Thus

$$
\psi_{g}^{C_{g} C_{0}}(x)=\int_{C} P_{C \cup C_{0}}(x, z)\left(g(z)-\psi_{g}^{C_{0}}(z)\right) d z .
$$

Therefore for $x \notin C \cup C_{0}$

$$
\psi_{\xi}^{C, C_{0}}(x)=\int_{C} P_{C \cup C_{0}}(x, z) \xi(z) d z-\int_{C} P_{C \cup C_{0}}(x, z) \psi_{\xi}^{C_{0}}(z) d z,
$$

and by (2.2) and (2.8)

$$
\begin{aligned}
\int\left|\psi_{\xi}^{C, C_{0}}(x)\right|^{2} d \mu(\xi) \leqq & 2 c \int_{C} \int_{C} P_{C \cup C_{0}}(x, z) G_{m}(z-\tilde{z}) P_{C \cup C_{0}}(x, \tilde{z}) d z d \tilde{z} \\
& +2 c \int_{C} \int_{C} P_{C \cup C_{0}}(x, z) K_{C_{0}}(z, \tilde{z}) P_{C \cup C_{0}}(x, \tilde{z}) d z d \tilde{z} .
\end{aligned}
$$

It follows from (2.33) that if $d(x, C) \geqq 1$ then

$$
E\left(\left|\psi_{\xi}^{C, C_{0}}(x)\right|^{2}\right) \leqq a e^{-m^{\prime} d(x, C)}, \quad m^{\prime}<m,
$$

for some constant $a$.

Hence we get the following lemma.

Lemma 2.5. Let $\xi(x)$ be a regular random field on $\mathbb{R}^{2}$ so that

$$
E\left(|\langle\varphi, \xi\rangle|^{2}\right) \leqq c \iint \varphi(x) \varphi(y) G_{m}(x-y) d x d y
$$

for $\varphi \in S\left(\mathbb{R}^{2}\right)$. Let $C_{0}$ and $C$ be two piecewise $C^{1}$-curves in $\mathbb{R}^{2}$ and let

$$
\psi_{\xi}^{C, C_{0}}(x)=\psi_{\xi}^{C \cup C_{0}}(x)-\psi_{\xi}^{C_{0}}(x)
$$


for $x \notin C \cup C_{0}$, where $\psi_{g}^{C}(x)$ is the solution of $\left(-\Delta+m^{2}\right) \psi=0$ in $\mathbb{R}^{2}-C$ and $\psi=g$ on $C$. Then there is a constant a depending only on $c$ and $m$ such that for $x \in \mathbb{R}^{2}-C \cup C_{0}$ with $d(x, C) \geqq 1$ we have

$$
E\left(\left|\psi_{\xi}^{C, C_{o}}(x)\right|^{2}\right) \leqq a e^{-m^{\prime} d(x, C)}, \quad m^{\prime}<m
$$

and if $\Lambda$ is a compact subset of $\mathbb{R}^{2}-C \cup C_{0}$ such that $d(\Lambda, C) \geqq 1$ then

$$
E\left(\int_{\Lambda}\left|\psi_{\xi}^{C, C_{0}}(x)\right|^{2} d x\right) \leqq a e^{-m^{\prime} d(\Lambda, C)} .
$$

Now in the same way as Theorem 2.1 follows from Lemma 2.2 we get the following theorem from Lemma 2.5.

Theorem 2.2. Let the notations be as in Theorem 2.1. Let $C_{0}$ be any fixed $C^{1}$-curve and let $C_{n}$ be any sequence of piecewise $C^{1}$-curves in $\mathbb{R}^{2}$ which tends to infinity in the sense that $d\left(0, C_{n}\right) \rightarrow \infty$. Let $\Lambda_{n}$ be any sequence of bounded sets in $\mathbb{R}^{2}$ such that $\Lambda_{n} \subset \mathbb{R}^{2}-C_{0} \cup C_{n}$ and $d\left(\Lambda_{n}, C_{n}\right) \rightarrow \infty$ as $n \rightarrow \infty$. Then for any $\alpha<m$ there is $a$ subsequence $n^{\prime}$ such that

$$
e^{\alpha / 2 d\left(\Lambda_{n^{\prime}}, C_{n^{\prime}}\right)} \sup _{x \in A_{n^{\prime}}}\left|\psi_{\xi}^{C_{0} \cup C_{n^{\prime}}}(x)-\psi_{\xi}^{C_{0}}(x)\right| \rightarrow 0
$$

for $\mu$-almost all $\xi$.

\section{Euclidean Markov Fields}

The free Euclidean quantum field of mass $m>0$ in $\mathbb{R}^{2}([1])$ is the tempered random field $\xi$ given by

$$
E\left(e^{i\langle\varphi, \xi\rangle}\right)=e^{-1 / 2 E_{m}(\varphi)}
$$

Hence $\xi$ is Gaussian and since

$$
E\left(|\langle\varphi, \xi\rangle|^{2}\right)=E_{m}(\varphi)
$$

we have that $\xi$ is a regular random field. Let now $C$ be a piecewise $C^{1}$-curve and let $\psi_{g}^{C}(x)$ be as defined in (2.3). Then $\xi \rightarrow \psi_{\xi}^{C}(x)$ is $\mu_{0}$-measurable, where $\mu_{0}$ is the probability measure corresponding to the free Euclidean quantum field of mass $m$. It is easily seen that the $\sigma$-algebra $B_{C}$ is generated by the functions $\xi \rightarrow \psi_{\xi}^{C}(x)$ for $x \notin C$. We have

$$
E\left(\psi_{\xi}^{C}(x) \psi_{\xi}^{C}(y)\right)=\int_{C} \int_{C} P_{C}\left(x, z_{1}\right) G_{m}\left(z_{1}-z_{2}\right) P_{C}\left(y, z_{2}\right) d z_{1} d z_{2} .
$$

Now from (2.4) we have that

$$
\int_{C} P_{C}\left(x, z_{1}\right) G_{m}\left(z_{1}-z_{2}\right) d z_{1}
$$

for $z_{1} \in C$ is the solution of (2.3), but this however is equal to $G_{m}\left(x-z_{2}\right)$, hence

$$
E\left(\psi_{\xi}^{C}(x) \psi_{\xi}^{C}(y)\right)=\int_{C} G_{m}(x-z) P_{C}(y, z) d z
$$


and we get

$$
E\left(\psi_{\xi}^{C}(x) \psi_{\xi}^{C}(y)\right)=E\left(\xi(x) \psi_{\xi}^{C}(y)\right) .
$$

Therefore if we set $\xi^{C}(x)=\xi(x)-\psi_{\xi}^{C}(x)$ then

$$
E\left(\xi^{C}(x) \psi_{\xi}^{C}(y)\right)=0
$$

for all $x$ and $y$ in $\mathbb{R}^{2}-C$. By definition $\psi_{\xi}^{C}(x)=\xi(x)$ for $x \in C$ so that $\xi^{C}(z)=0$ for $z \in C$. Since both $\xi^{C}(x)$ and $\psi_{\xi}^{C}(y)$ are Gaussian random fields with zero expectation we see that $\xi^{C}(x)$ and $\psi_{\xi}^{C}(y)$ are stochastically independent. An easy computation gives

$$
E\left(\xi^{C}(x) \xi^{C}(y)=G_{m}^{C}(x, y)\right.
$$

where $G_{m}^{C}(x, y)$ is the kernel of the operator $\left(-\Delta_{C}+m^{2}\right)^{-1}, \Delta_{C}$ being the Laplacian with Dirichlet boundary conditions on $C$, while

$$
E\left(\psi_{\xi}^{C}(x) \psi_{\xi}^{C}(y)\right)=G_{m}(x-y)-G_{m}^{C}(x, y) .
$$

Therefore

$$
\xi(x)=\xi^{C}(x)+\psi_{\xi}^{C}(x)
$$

is the splitting of the random field $\xi(x)$ into two mutually independent random fields. From this we get that if $E_{0}(\cdot \mid C)$ is the conditional expectation with respect to the measure $\mu_{0}$ and the $\sigma$-algebra $B_{C}$ then for any $f \in L_{1}\left(\mu_{0}\right)$

$$
E_{0}(f \mid C)(\eta)=E_{0}^{C}\left(f\left(\xi^{C}+\psi_{\eta}^{C}\right)\right),
$$

where $E_{0}^{C}$ is the expectation with respect to the random field $\xi^{C}(x)$. Let now $C$ be a piecewise $C^{1}$-curve such that $\mathbb{R}^{2}-C$ consists of two components $\Omega_{+}$and $\Omega_{-}$. In this case it is well known that if $x \in \Omega_{+}$and $y \in \Omega_{-}$then $G_{m}^{\mathrm{C}}(x, y)=0$ which implies that $\xi^{C}(x)$ for $x \in \Omega_{+}$is stochastically independent of $\xi^{C}(y)$ for $y \in \Omega_{-}$. But this together with (3.11) gives that if $f_{ \pm}$are $B_{\Omega_{ \pm}}$-measurable respectively and bounded then

$$
E\left(f_{+} f_{-} \mid C\right)(\eta)=E\left(f_{+} \mid C\right)(\eta) \cdot E\left(f_{-} \mid C\right)(\eta) .
$$

If (3.12) holds whenever $C$ is a piecewise $C^{1}$-curve such that $\mathbb{R}^{2}-C$ has two components $\Omega_{+}$and $\Omega_{-}$and $f_{+}$and $f_{-}$are bounded $B_{\Omega_{+}}$-respectively $B_{\Omega_{-}-}$ measurable functions we say that the corresponding random field has the global Markov property. If (3.12) holds whenever $C$ is a bounded piecewise $C^{1}$-curve such that $\mathbb{R}^{2}-C$ has two components $\Omega_{+}$and $\Omega_{-}$we say that the corresponding random field has the local Markov property. Thus the free Euclidean quantum field of positive mass in $\mathbb{R}^{2}$ has the global Markov property.

Let now, for each bounded Borel set $\Lambda \subset \mathbb{R}^{2}, a_{\Lambda}(\xi)$ be a $\mu_{0}$-measurable function. We say that $a_{\Lambda}$ is an additive functional of the free Euclidean quantum field $\xi$ iff $a_{A}(\xi)$ is $B_{A}$-measurable and

$$
a_{\Lambda_{1} \cup \Lambda_{2}}=a_{\Lambda_{1}}(\xi)+a_{\Lambda_{2}}(\xi)
$$

whenever $\Lambda_{1} \cap \Lambda_{2}=\emptyset$. 
Let $\mu_{0}^{C}(\xi \mid \eta)$ be the probability measure on $S^{\prime}\left(\mathbb{R}^{2}\right)$ corresponding to the random field $\xi^{C}(x)+\psi_{\eta}^{C}(x)$ for fixed $\eta \in S^{\prime}\left(\mathbb{R}^{2}\right)$. Then by (3.11)

$$
E_{0}(f \mid C)(\eta)=\int_{S^{\prime}\left(\mathbb{R}^{2}\right)} f(\xi) d \mu_{0}^{C}(\xi \mid \eta) .
$$

It follows easily that $\mu_{0}^{C}(\cdot \mid \eta)$ also has the global Markov property. Let us now assume that $a_{\Lambda}(\xi)=0$ for $\mu$-almost all $\xi$ if $|\Lambda|=0$, where $|\Lambda|$ is the Lebesgue measure of $\Lambda$. Let $C$ be any curve such that $\mathbb{R}^{2}-C$ has two components $\Omega_{+}$and $\Omega_{-}$and let $\Lambda$ be a fixed open bounded subset of $\mathbb{R}^{2}$ and set $\Lambda_{ \pm}=\Lambda \cap \Omega_{+}$. Since

$$
e^{-a_{\Lambda}}=e^{-a_{\Lambda}+} \cdot e^{-a_{\Lambda-}}
$$

we have, assuming that $\partial \Lambda$ is piecewise $C^{1}$, that

$$
d \mu_{a}^{i \Lambda}(\xi \mid \eta)=\left(E_{0}\left(e^{-a_{\Lambda}} \mid \partial \Lambda\right)(\eta)\right)^{-1} e^{-a_{\Lambda}} d \mu_{0}^{\hat{\partial} \Lambda}(\xi \mid \eta)
$$

again has the global Markov property, i.e. if $E_{a, \eta}^{\partial A}$ is the expectation with respect to the measure $\mu_{a}^{\partial \Lambda}(\cdot \mid \eta)$ and $E_{a, \eta}^{\partial A}(\cdot \mid C)$ is the corresponding conditional expectation with respect to the $\sigma$-algebra $B_{C}$, then

$$
E_{a, \eta}^{\partial \Lambda}\left(f_{+} f_{-} \mid C\right)=E_{a, \eta}^{\hat{i} \Lambda}\left(f_{+} \mid C\right) E_{a, \eta}^{\hat{\partial A}}\left(f_{-} \mid C\right),
$$

where $f_{+}$and $f_{-}$are $B_{\Omega_{+}}$- and $B_{\Omega_{-}}$-measurable respectively. From (3.14) and (3.16) we also get that if $\Lambda_{0} C \Lambda$ and $\partial \Lambda_{0}$ as well as $\partial \Lambda$ are piecewise $C^{1}$-curves and $f$ is $B_{A_{0}}$-measurable then

$$
E_{a, \eta}^{\hat{c} \Lambda}\left(f \mid \partial \Lambda_{0}\right)(\tilde{\eta})=E_{a, \tilde{\eta}}^{\partial \Lambda_{0}}(f) .
$$

If $f$ is $B_{\Lambda_{0}}$-measurable and $\Lambda_{0} \subset \Lambda$ we have that

$$
E_{a, \eta}^{\partial \Lambda}\left(E_{a, \eta}^{\partial \Lambda}\left(f \mid \partial \Lambda_{0}\right)\right)=E_{a, \eta}^{\hat{c} \Lambda}(f)
$$

by the property of the conditional expectation. From (3.19) and (3.18) we get that if $f$ is $B_{\Lambda_{0}}$-measurable then if $\Lambda_{0} \subset \Lambda$ such that $\partial \Lambda_{0}$ as well as $\partial \Lambda$ are piecewise $C^{1}$ then

$$
\int f(\xi) d \mu_{a}^{\partial \Lambda}(\xi \mid \eta)=\iint f(\xi) d \mu_{a}^{\hat{\alpha} \Lambda_{0}}(\xi \mid \tilde{\eta}) d \mu_{a}^{\hat{i} \Lambda}(\tilde{\eta} \mid \eta)
$$

or

$$
\mu_{a}^{\hat{\partial \Lambda}}(\xi \mid \eta)=\int \mu_{a}^{\partial \Lambda_{0}}(\xi \mid \tilde{\eta}) d \mu_{a}^{\hat{\delta} \Lambda}(\tilde{\eta} \mid \eta)
$$

Remark now that if $\Lambda_{0}$ is compactly contained in $\Lambda$ then $\mu_{0}^{\delta \Lambda}(\cdot \mid \eta)$ restricted to $B_{\Lambda_{0}}$ is absolutely continuous with respect to the restriction of $\mu_{0}$ to $B_{A_{0}}$, hence also the restriction to $B_{A_{0}}$ of $\mu_{a}^{\partial A}(\cdot \mid \eta)$ is absolutely continuous with respect to $\mu_{0}$. Let now $P_{\Lambda_{0}}^{a}(\Lambda)$ be the set of probability measures on $B_{\Lambda_{0}}$ of the form

$$
\int \mu_{a}^{\partial \Lambda}(\xi \mid \eta) \varrho(\eta) d \mu_{0}(\eta)
$$

where $\varrho(\eta)$ is an arbitrary positive normalized function in $L_{1}\left(\mu_{0}\right)$. From (3.21) it follows that $P_{\Lambda_{0}}^{a}(\Lambda)$ is a decreasing function of $\Lambda$ and set

$$
P_{\Lambda_{0}}^{a}=\bigcap_{\Lambda} P_{A_{0}}^{a}(\Lambda)
$$


It follows from the definition of $P_{\Lambda_{0}}^{a}$ that if $\Lambda_{0} \subset \Lambda_{1}$ then the elements in $P_{A_{0}}^{a}$ are restrictions to $B_{A_{0}}$ of elements in $P_{A_{1}}^{a}$. Hence there is a unique set of measures $G^{a}$ such that the restriction to $B_{A_{0}}$ of the elements in $G^{a}$ are the elements in $P_{A_{0}}^{a}$. The elements in $G^{a}$ are called the Gibbsmeasures given by the additive functional $a$.

It is easily seen that the elements in $P_{\Lambda_{0}}^{\alpha}(\Lambda)$ have the Markov property with respect to curves $C \subset A_{0}$ such that $\mathbb{R}^{2}-C$ consists of two components $\Omega_{+}$and $\Omega_{-}$, where $\Omega_{-}$is the bounded component. The Markov property (3.12) may also be written in the form

$$
E_{\mu}\left(f \mid \Omega_{+}\right)=E_{\mu}(f \mid C)
$$

whenever $f$ is $B_{\Omega_{-}}$-measurable, where now $E_{\mu}$ is the expectation with expect to a measure $\mu$ of the form (3.22). In fact (3.24) follows immediately from the Markov property of $\mu_{a}^{\partial A}(\cdot \mid \eta)$ and (3.22). This proves the following theorem

Theorem 3.1. The Gibbsmeasures given by an additive functional $a_{A}$ of the free Euclidean field of positive mass in $\mathbb{R}^{2}$ have the local Markov property.

Remark 1. This theorem was first proved by Newman [8].

Remark 2. Assume now that the additive functional $a_{A}(\xi)$ is Euclidean invariant i.e. $a_{g \Lambda}(\xi)=a_{\Lambda}\left(g^{-1} \xi\right)$ for any Euclidean transformation $g$. There are then two interesting questions concerning the Gibbsmeasures $G^{a}$ given by the additive functional $a$.

1. When is there exactly one element in $G^{a}$ ?

2. When are the elements in $G^{a}$ globally Markov?

We say that $\mu \in G^{a}$ is an extreme phase if there are no other elements in $G^{a}$ which are absolutely continuous with respect to $\mu$. It is easily seen that if $v \in G^{a}$ and $v$ is absolutely continuous with respect to $\mu$ then $d v=\varrho d \mu$ where $\varrho$ is $B_{\mathbb{R}^{2}-A^{-}}^{\mu}$ measurable for any open bounded set $\Lambda$, where $B_{\mathbb{R}^{2}-\Lambda}^{\mu}$ is the $\sigma$-algebra generated by $B_{\mathbb{R}^{2}-A}$ and the $\mu$-null sets. Hence $\varrho$ is $B_{\infty}^{\mu}$-measurable with $B_{\infty}^{\mu}=\bigcap_{\Lambda} B_{\mathbb{R}^{2}-\Lambda}^{\mu}$. So that $\mu$ is an extreme phase if and only if $\mu \uparrow B_{\infty}^{\mu}$ is a trivial probability measure. Since

$$
E_{\mu}\left(\cdot \mid B_{\mathbb{R}^{2}-\Lambda}^{\mu}\right) \rightarrow E_{\mu}\left(\cdot \mid B_{\infty}^{\mu}\right)
$$

monotonously as $\Lambda \mathbb{R}^{2}$ and strongly as projections in $L_{2}(\mu)$ we have that $\mu \nmid B_{\infty}^{\mu}$ is trivial if and only if $E_{\mu}\left(\cdot \mid B_{\mathbb{R}^{2}-A}^{\mu}\right) \rightarrow E_{\mu}(\cdot)$ as $\Lambda \mathbb{R}^{2}$. We therefore have the following theorem

Theorem 3.2. $\mu \in G^{a}$ is an extreme phase if and only if

$$
E_{\mu}\left(\cdot \mid B_{\mathbb{R}^{2}-\Lambda}^{\mu}\right) \rightarrow E_{\mu}(\cdot)
$$

as $\Lambda / \mathbb{R}^{2}$, where $\Lambda$ ranges through the filter of bounded open subsets of $\mathbb{R}^{2}$. 


\section{The Trigonometric Interaction}

The trigonometric interaction $[21,4,22,23]$ is obtained by considering the additive functional $U_{A}(\xi)$ of the form

$$
U_{\Lambda}(\xi)=\int_{\Lambda}: \cos \left(\alpha \xi(x)+\theta_{0}\right): d x
$$

with $\alpha^{2}<4 \pi$. Fröhlich proved [22] that $e^{-\lambda U_{\Lambda} \in L_{1}}\left(\mu_{0}\right)$ for all $\lambda$ and moreover that $G^{\lambda U}$ is non empty, where $G^{\lambda U}$ is the set of Gibbsmeasures given by the Euclidean invariant additive functional $\lambda U_{A}(\xi)$. Fröhlich and Seiler [4] proved that there is a $\mu_{\lambda} \in G^{\lambda U}$ such that $\mu_{\lambda}$ is weakly analytic in $\lambda$ for $|\lambda|<\lambda_{0}$, where $\lambda_{0}$ depends only on $\alpha$ and on $m_{0}$, the mass of the free field. This result is proven by using the cluster expansion which was first introduced by Glimm et al. [2] to prove the existence of the infinite volume limit for the weak polynomial interaction.

It follows from the result of Fröhlich and Seiler that for $|\lambda|<\lambda_{0}$ we have

$$
E_{\lambda}\left(|\langle\varphi, \xi\rangle|^{2}\right) \leqq C^{\prime} E_{m}(\varphi)
$$

for some positive $m>0$, hence by the remark following (2.2), also for any $m$. Thus we have

$$
E_{\lambda}\left(|\langle\varphi, \xi\rangle|^{2}\right) \leqq C E_{m_{0}}(\varphi)
$$

for any $\varphi \in S\left(\mathbb{R}^{2}\right)$, where $E_{\lambda}$ is the expectation with respect to $\mu_{\lambda}$ and $m_{0}$ is the mass of the free Euclidean field corresponding to the probability distribution $\mu_{0}$ on $S^{\prime}\left(\mathbb{R}^{2}\right)$. From (4.3) we have

Lemma 4.1. Let $\mu_{\lambda}$ be the Gibbsmeasure corresponding to the additive functional

$$
\lambda U_{\Lambda}=\lambda \int_{\Lambda}: \cos \left(\alpha \xi(x)+\theta_{0}\right): d x
$$

with $\alpha^{2}<4 \pi$ which is analytic in $\lambda$ for $|\lambda|<\lambda_{0}$. Then $\mu_{\lambda}$ defines a regular tempered random field on $\mathbb{R}^{2}$.

Following Fröhlich and Seiler [4] we introduce for $\theta \in[0,2 \pi)$ the random fields

$$
c_{\theta}(x)=: \cos (\alpha \xi(x)+\theta):
$$

and set

$$
c_{\theta}(f)=\int c_{\theta}(x) f(x) d x
$$

for $f \in C_{0}\left(\mathbb{R}^{2}\right)$. Then $c_{\theta}(f) \in L_{2}\left(\mu_{0}\right)$ where $\mu_{0}$ is the probability measure on $S^{\prime}\left(\mathbb{R}^{2}\right)$ corresponding to the free field of mass $m_{0}$. Set $\{x, \theta\}_{N}=\left\{x_{1}, \ldots, x_{N} ; \theta_{1}, \ldots, \theta_{N}\right\}$ and

$$
S^{\lambda}\left(\{x, \theta\}_{N}\right)=E_{\lambda}\left(\prod_{j=1}^{N} c_{\theta_{j}}\left(x_{j}\right)\right) .
$$

Set also

$$
S_{\Lambda}^{\lambda}\left(\{x, \theta\}_{N}\right)=E_{0}\left(e^{-\lambda U_{\Lambda}}\right)^{-1} E_{0}\left(\prod_{j=1}^{N} c_{\theta_{j}}\left(x_{j}\right) e^{-\lambda U_{\Lambda}}\right)
$$


and set

$$
S_{\Lambda}^{\lambda}\left(x_{1}, \ldots, x_{N}\right)=E_{0}\left(e^{-\lambda U_{\Lambda}}\right)^{-1} E_{0}\left(\prod_{j=1}^{N} \xi\left(x_{j}\right) e^{-\lambda U_{\Lambda}}\right),
$$

then an integration by parts with respect to the normal measure $\mu_{0}$ (see [4]) shows that $S_{\Lambda}^{\lambda}\left(x_{1}, \ldots, x_{n}\right)$ may be expressed by $S_{\Lambda}^{\lambda}\left(\{x, \theta\}_{N}\right)$. Thus the convergence of $S_{\Lambda}^{\lambda}\left(\{x, \theta\}_{N}\right)$ to $S^{\lambda}\left(\{x, \theta\}_{N}\right)$ implies the weak convergence

$$
\left(\int e^{-\lambda U_{\Lambda}(\xi)} d \mu_{0}\right)^{-1} e^{-\lambda U_{\Lambda}(\xi)} d \mu_{0}(\xi) \rightarrow d \mu_{\lambda}(\xi),
$$

as $\Lambda \nearrow \mathbb{R}^{2}$.

The convergence of (4.6) to (4.5) is proven by Fröhlich and Seiler [4] by showing that the cluster expansion converges. The cluster expansion is obtained in the following way as described in [4]. We keep concepts and notation as close as possible to those used in [4].

First one covers $\mathbb{R}^{2}$ by a square lattice $L$ with lattice constant 1 . Let $B$ denote the set of all bonds i.e. square sides in $L$. Let $C_{B}$ be the operator $\left(-\Delta_{B}+m^{2}\right)^{-1}$, where $\Delta_{B}$ is the Laplacian with Dirichlet boundary conditions on $B$. The Gaussian measure on $S^{\prime}\left(\mathbb{R}^{2}\right)$ with mean zero and covariance $C_{B}$ is denoted by $\mu_{B}$ and let $\langle\cdot\rangle_{C_{B}}$ be the expectation with respect to $\mu_{B}$. The measure $\mu_{B}$ decouples regions that are separated by bonds of $B$ completely in the sense that if $\varphi$ and $\psi$ in $S\left(\mathbb{R}^{2}\right)$ are such that $\operatorname{supp} \varphi$ is separated from $\operatorname{supp} \psi$ by a closed line of bonds in $B$ then $\langle\psi, \xi\rangle$ and $\langle\varphi, \xi\rangle$ are independent with respect to the probability measure $\mu_{B}$. Let now

$$
S_{\Lambda, B}^{\lambda}\left(\{x, \theta\}_{N}\right)=\left\langle e^{-\lambda U_{A}}\right\rangle_{C_{B}}^{-1}\left\langle\prod_{j=1}^{N} c_{\theta_{j}}\left(x_{j}\right) e^{-\lambda U_{A}}\right\rangle_{C_{B}} .
$$

It is obvious that (4.9) is independent of $\Lambda$ as soon as $\Lambda$ contains the smallest union of lattice squares of $L$ containing $\{x\}_{N}$. Therefore the limit of (4.9) as $\Lambda \nearrow \mathbb{R}^{2}$ exists trivially.

The cluster expansion is obtained by removing the Dirichlet condition on the bonds of $B$ step by step and estimating after a partial resummation the terms in the final series. Now removing the Dirichlet condition on a bond $b \in B$ introduces a convergence factor proportional to $m_{0}^{-\eta}(\eta>0)$ or, in a term localized near $x$, a convergence factor proportional to $e^{-m_{0} d(b, x)}$. These factors yield the convergence of the expansion. For details on the procedure see [2] and [4]. Following [4] we introduce the following notations.

A collection of bonds $b$ in $B$ is denoted by $\Gamma$ and let $\Gamma^{c}=B-\Gamma$ (the completement of $\Gamma$ in $B$ ). Let $\left.w\{x\}_{N}\right)$ be a function of compact support in $L_{p}\left(\mathbb{R}^{2 N}\right)$ with $p(p-1)^{-1} \alpha^{2}<4 \pi$. Set $X_{0}=\operatorname{supp} w$. Let $X$ range over finite unions of closed lattice squares and let $\Gamma$ range over the set of finite collections of bonds in $B$ such that

(i) each connected component of $X-\Gamma^{c}$ meets $X_{0}$

(ii) $\Gamma \subset \operatorname{Int} X$. 
Set

$$
C\left(\{s\}_{B}\right)=\sum_{\Gamma \subset B} \prod_{b \in \Gamma} s_{b} \prod_{b \in \Gamma^{c}}\left(1-s_{b}\right) C_{\Gamma^{c}}
$$

where $C_{\Gamma^{\mathrm{c}}}$ is the operator $\left(-\Delta_{\Gamma^{\mathrm{c}}}+m_{0}^{2}\right)^{-1}$ and $\Delta_{\Gamma^{\mathrm{c}}}$ is the Laplacian with Dirichlet boundary conditions on $\Gamma^{\mathrm{c}}$. [Clearly $C(1,1, \ldots)=\left(-\Delta+m_{0}^{2}\right)^{-1}$.] Let

$$
\begin{aligned}
s(\Gamma) & =\left\{s(\Gamma)_{b}\right\}_{b \in B} \text { with } \\
s\left(\Gamma_{b}\right) & =\left\{\begin{array}{l}
s_{b}, b \in \Gamma \\
0, b \notin \Gamma .
\end{array} \quad\left(0 \leqq s_{b} \leqq 1\right)\right.
\end{aligned}
$$

Expectations with respect to the Gaussianmeasure on $S^{\prime}\left(\mathbb{R}^{2}\right)$ with mean 0 and covariance $C(\cdot)$ are denoted $\langle\cdot\rangle_{C(\cdot)}$ and we set

$$
Z_{\Gamma}^{\lambda}(\Lambda)=\left\langle e^{-\lambda U_{\Lambda}}\right\rangle_{C_{\Gamma}} .
$$

The cluster expansion is summarized in the equation

$$
\begin{aligned}
S_{\Lambda}^{\lambda}\left(w,\{\theta\}_{N}\right)= & \sum_{X, \Gamma} \int_{0}^{1} \ldots \int_{0}^{1} \prod_{b \in \Gamma} d s_{b} \frac{\partial}{\partial s_{b}}\left\langle\int \prod_{j=1}^{N} c_{\theta_{j}}\left(x_{j}\right) d x_{j}\right. \\
& \left.\left.\cdot w\{x\}_{N}\right) e^{-\lambda U_{\Lambda} \cap x(\xi)}\right\rangle_{C(s(\Gamma))} Z_{\partial X}^{\lambda}(\Lambda-X) Z^{\lambda}(\Lambda)^{-1} .
\end{aligned}
$$

The cluster expansion (4.14) is derived in [2, Sect. 3, Eq. (3.15)]. Now the important property of (4.14) utilized by Fröhlich and Seiler is that it is model independent. The basic result of [2] is the following theorem.

Theorem 4.1 [2, Sect. 4]. The convergence of the cluster expansion (4.14) implies the convergence (4.8) and also the exponential cluster property.

For the proof of this theorem for $P(\varphi)_{2}$-models see [2].

The convergence of the cluster expansion follows from three essential estimates which are given here as the three following lemmas and which correspond to Proposition 5.1, 5.2 and 5.3 of [2].

Lemma 4.2 [2] (Proposition 5.1). The number of terms in the cluster expansion (4.14) with a fixed value of $|X|$ is bounded by $C_{1} e^{K_{1}|X|}\left(K_{1}=19\right)$.

This lemma is entirely model independent and is therefore proved in [2].

Lemma 4.3 [2] (Proposition 5.2). There is a $K_{2}$ independent of $\lambda, \Lambda$ as long as $|\lambda| \leqq \lambda_{1}(\alpha)$ such that $\left|Z_{\partial X}^{\lambda}(\Lambda-X) Z^{\lambda}(\Lambda)^{-1}\right| \leqq e^{K_{2}|X|}$.

Proof. This follows from the proof of Proposition 5.2 [2] and the basic estimate

$$
\frac{1}{2} \leqq\left|Z^{\lambda}(\Delta)\right| \leqq \frac{3}{2}
$$

which is given in (2.4) of [2].

The third basic estimate is 
Lemma 4.4 [2] (Proposition 5.3), [4] (Proposition 2.2). There is a constant $K_{3}$ and a norm $|w|$ on test functions such that for any $K>0$, for any $\Lambda$ and for any $\lambda$ with $|\lambda|<\lambda_{1}(\alpha)$ we have for all $m_{0}>\mu_{K}$ that

$$
\begin{aligned}
& \left|\left\langle\int \partial^{\Gamma} \int \prod_{i=1}^{N} c_{\theta_{t}}\left(x_{i}\right) e^{-\lambda U_{\Lambda}(\xi)} d \mu_{C(s(\Gamma))}(\xi) d s(\Gamma), w\right\rangle\right| \\
& \quad \leqq e^{-K|\Gamma|+K_{3}|\Lambda|}|w| .
\end{aligned}
$$

Moreover if $w$ is of compact support $X$ then we may take $|w|=C_{N, X} \cdot\|w\|_{\infty}$.

This lemma is [2] (Proposition 2.2) and for the proof we refer the reader to the original paper [2].

Let us now write the cluster expansion (4.14) in the form

$$
S_{\Lambda}^{\lambda}\left(w,\{\theta\}_{N}\right)=\sum_{X, \Gamma} \int T^{\lambda}\left(\{x\}_{N},\{\theta\}_{N}, \Lambda, X, \Gamma\right) w\left(\{x\}_{N}\right) d\{x\}_{N},
$$

with

$$
\{x\}_{N}=\left(x_{1}, \ldots, x_{N}\right), d\left\{x_{N}\right\}=\prod_{j=1}^{N} d x_{j} .
$$

We have then the following lemma which corresponds to Theorem 4.1 of [2].

Lemma 4.5. Let $K>0$ be given. Then there is a constant $\mu_{K}$ depending on $K$ such that for $|\lambda|<\lambda_{1}(\alpha)$ and for $m_{0}>\mu_{K}$ there is an $S$-norm $|\cdot|$ such that

$$
\sum_{\substack{X, I \\|X| \geqq D}}\left|\int T^{\lambda}\left(\{x\}_{N},\{\theta\}_{N}, \Lambda, X, \Gamma\right) w\left(\{x\}_{N}\right) d\{x\}_{N}\right| \leqq|w| e^{-K(D-N)} .
$$

Moreover if $w$ is of compact support then we may take $|w|=C_{N, X}\|w\|_{\infty}$ where $X=\operatorname{supp} w$.

Proof. The proof is the proof of [2] (Theorem 4.1) and goes as follows. We replace $\Lambda$ by $\Lambda \cap X$ in Lemma 4.4. For $X$ in (4.16) we have $X=\bigcup_{i} \bar{X}_{i}$ with $r \leqq N$ and $X_{i}$ connected. Moreover

$$
\Gamma \subset \bigcup_{i=1}^{r} \operatorname{Int} \bar{X}_{i}
$$

and $\bar{X}_{i}-\Gamma^{c}=X_{i}$. Thus

$$
\left|X_{i}\right|-1 \leqq 2\left|\Gamma \cap \operatorname{Int} \bar{X}_{i}\right| \text { and }|X|-N \leqq 2|\Gamma| .
$$

Hence we replace the upper bound in Lemma 4.4 by

$$
e^{-K(|X|-N)}|w|
$$

with a new choice of $K$ and $|w|$. Lemma (4.5) follows directly by combining (4.19) with Lemma 4.2, Lemma 4.3.

We shall need the following two lemmas which are a special case of a result due to Guerra, Rosen and Simon [16] called "conditioning". 
Lemma 4.6. Let $\chi_{1}$ and $\chi_{2}$ be bounded positive functions on $\mathbb{R}^{2}$ and let $C\left(\chi_{i}\right)$ $=\left(-\Delta+m_{0}^{2}+\chi_{i}\right)^{-1}$, then if $\chi_{1} \geqq \chi_{2} \geqq-m_{0}^{2}+\varepsilon, \varepsilon>0$ we have, for arbitrary $f$ and $g$ in $C\left(\mathbb{R}^{2}\right) \cap L_{1}\left(\mathbb{R}^{2}\right)$, that

$$
\begin{aligned}
& \left\langle\exp \left\{\int(f(x): \cos \alpha \xi(x):+g(x): \sin \alpha \xi(x):) d x\right\}\right\rangle_{C\left(\chi_{1}\right)} \\
& \quad \leqq\left\langle\exp \left\{\int(f(x): \cos \alpha \xi(x):+g(x): \sin \alpha \xi(x):) d x\right\}\right\rangle_{C\left(\chi_{2}\right)}
\end{aligned}
$$

Proof. Let $a=C\left(\chi_{1}\right)$ and $b=C\left(\chi_{2}\right)-C\left(\chi_{1}\right)$ and let $\xi_{a}(x)$ and $\xi_{b}(x)$ be Gaussian random fields with mean zero and covariance $a$ and $b$ respectively. Then $\xi(x)$ $=\xi_{a}(x)+\xi_{b}(x)$ has covariance $C\left(\chi_{2}\right)$. Thus by Jensen's inequality we have

$$
\begin{aligned}
\langle\exp & \left\{\int(f(x): \cos \alpha \xi(x):+g(x): \sin \alpha \xi(x):) d x\right\rangle_{C\left(x_{2}\right)} \\
= & \left\langle\operatorname { e x p } \left\{\int \left( f(x): \cos \alpha\left(\xi_{a}(x)+\xi_{b}(x)\right):+g(x)\right.\right.\right. \\
& \left.\left.: \sin \alpha\left(\xi_{a}(x)+\xi_{b}(x)\right):\right) d x\right\rangle_{a, b} \\
& \geqq\left\langle\operatorname { e x p } \left\{\int \left( f(x)\left\langle: \cos \alpha\left(\xi_{a}(x)+\xi_{b}(x)\right):\right\rangle_{b}+g(x)\right.\right.\right. \\
& \left.\left.\left.\left\langle\sin \alpha\left(\xi_{a}(x)+\xi_{b}(x)\right):\right\rangle_{b}\right) d x\right\}\right\rangle_{a} \\
= & \left\langle\exp \left\{\int\left(f(x): \cos \alpha \xi_{a}(x):+g(x): \sin \alpha \xi_{a}(x):\right) d x\right\}\right\rangle_{a}
\end{aligned}
$$

(where \langle\rangle$_{a, b}$ means integration with respect to $\xi_{a}$ and $\xi_{b}$, and \langle\rangle$_{a},\langle\rangle_{b}$ means integration with respect to $\xi_{a}$ resp. $\xi_{b}$ alone), since

$$
: \cos \alpha\left(\xi_{a}+\xi_{b}\right):=: \cos \alpha \xi_{a}:: \cos \alpha \xi_{b}:-: \sin \alpha \xi_{a}:: \sin \alpha \xi_{b}:
$$

and

$$
: \sin \alpha\left(\xi_{a}+\xi_{b}\right):=: \sin \alpha \xi_{a}:: \cos \alpha \xi_{b}:+: \sin \alpha \xi_{b}:: \cos \alpha \xi_{a}:
$$

and

$$
\left\langle: \cos \alpha \xi_{b}:\right\rangle_{b}=1 \text {, while }\left\langle: \sin \alpha \xi_{b}:\right\rangle_{b}=0 .
$$

This proves the lemma.

Lemma 4.7. Let $\gamma_{1}$ and $\gamma_{2}$ be two piecewise smooth curves in $\mathbb{R}^{2}$ and let $C\left(\gamma_{i}\right)$ $=\left(-\Delta_{\gamma_{i}}+m_{0}^{2}\right)^{-1}$, where $\Delta_{\gamma_{i}}$ is the Laplacian with Dirichlet boundary conditions on $\gamma_{i}$. Then if $\gamma_{1} \supset \gamma_{2}$ we have that, for arbitrary $f$ and $g$ in $C\left(\mathbb{R}^{2}\right) \cap L_{1}\left(\mathbb{R}^{2}\right)$,

$$
\begin{aligned}
& \left\langle\exp \left\{\int(f(x): \cos \alpha \xi(x):+g(x): \sin \alpha \xi(x):) d x\right\}\right\rangle_{C\left(\gamma_{1}\right)} \\
& \quad \leqq\left\langle\exp \left\{\int(f(x): \cos \alpha \xi(x):+g(x): \sin \alpha \xi(x):) d x\right\}\right\rangle_{C\left(\gamma_{2}\right)} .
\end{aligned}
$$

Proof. We may approximate in the strong operator sense $\left(-\Delta_{\gamma_{2}}+m_{0}^{2}\right)^{-1}$ by $\left(-\Delta+m_{0}^{2}+\chi_{i, n}\right)^{-1}$, where $\chi_{i, n}, i=1,2$ are smooth positive functions going to 0 as $n \rightarrow \infty$. Since $\gamma_{1} \supset \gamma_{2}$ it is easy to see that this approximation may be done in such a way that $\chi_{1, n} \geqq \chi_{2, n}$. From the strong convergence of the covariances follows weak convergence of the corresponding Gaussian measures. Hence the inequality of this lemma follows from that of Lemma 4.6.

For our purpose we need a stronger version of Theorem 4.1 and to get this we shall need a stronger version of Lemma 4.3,4.4, and 4.5. Let us first introduce the 
notation

$$
c_{\varphi}^{\Lambda}(f)=\int_{\Lambda} c_{\varphi}(x) f(x) d x
$$

for $f \in L_{2}\left(\mathbb{R}^{2}\right)$, where $c_{\varphi}(x)=: \cos \alpha \xi(x)+\varphi:$. Then

$$
\int\left|c_{\varphi}^{\Lambda}(f)\right|^{2} d \mu_{0}=\int_{\Lambda} \int_{\Lambda} e^{\alpha^{2} G_{m_{0}}(x-y)} f(x) f(y) d x d y
$$

which is finite since $\Lambda$ is bounded and $\alpha^{2}<4 \pi, G_{m_{0}}(x-y) \sim \frac{1}{2 \pi}|\ln (x-y)|$, so that $e^{\alpha^{2} G_{m_{0}}(x-y)}$ is a bounded integral kernel in $L_{2}\left(\mathbb{R}^{2}\right)$. For this reason we have that $f \rightarrow c_{\varphi}^{\Lambda}(f)$ is a bounded map from $L_{2}\left(\mathbb{R}^{2}\right)$ into $L_{2}\left(d \mu_{0}\right)$. Let now $f_{i} \in C(\Lambda)$ such that

$$
\left|f_{i}(x)\right| \leqq 2 e^{\alpha^{2} / 2 K_{i \Lambda}(x, x)}
$$

where $K_{\partial \Lambda}(x, y)$ is given by (2.10). We have that

$$
\left|K_{\partial \Lambda}(x, x)\right| \leqq \frac{1}{2 \pi}|\ln d(x, \partial \Lambda)| e^{-m_{0} d(x, \partial \Lambda)}
$$

so that $\left|f_{i}(x)\right|^{2} \leqq 4|d(x, \partial \Lambda)|^{\left(-\left(\alpha^{2}\right) / 2 \pi\right)}$ and hence for $\alpha^{2}<2 \pi$ we have that (4.21) implies that $f_{i} \in L_{2}\left(\mathbb{R}^{2}\right)$.

Let now $f_{i} \in C(\Lambda), \Lambda$ open and bounded, satisfy $(4.21), \varphi_{i} \in[0,2 \pi), i=1, \ldots, k$ for some fixed $k$. Then by Lemma 4.7 we have

$$
\left\langle e^{-\lambda\left(U_{\Lambda}+\sum_{i=1}^{k} c_{\varphi_{i}}^{\Lambda}\left(f_{i}\right)\right)}\right\rangle_{C(\partial X)} \leqq\left\langle e^{\left.-\lambda\left(U_{\Lambda}+\sum_{i=1}^{k} c_{\varphi_{1}}^{A}\left(f_{i}\right)\right)\right\rangle_{0}}\right.
$$

where \langle\rangle$_{0}$ is the expectation with respect to $\mu_{0}$.

Thus, with $\tilde{U}_{\Lambda}=U_{\Lambda}+\sum_{i=1}^{k} c_{\varphi_{i}}^{\Lambda}\left(f_{i}\right)$

$$
\left\langle e^{-\lambda \tilde{U}_{A}}\right\rangle_{0}^{-1}\left\langle e^{-\lambda \tilde{U}_{A-X}}\right\rangle_{C(\partial X)} \leqq\left\langle e^{-\lambda \tilde{U}_{X}}\right\rangle_{C(\partial X)}^{-1}
$$

Let now $\Gamma(X)$ be all the lattice bounds in $X$, then again by Lemma 4.7 we have

$$
\left\langle e^{-\lambda \tilde{U}_{X}}\right\rangle_{C(\hat{O} X)} \leqq\left\langle e^{-\lambda \tilde{U}_{X}}\right\rangle_{C(\Gamma(X))}=\prod_{\Delta_{\imath} \subset X}\left\langle e^{-\lambda \tilde{U}_{\Delta_{i}}}\right\rangle_{\partial \Delta_{\imath}}
$$

where $\Delta_{i}$ are the unit squares of the lattice. Since $k$ is fixed and $f_{j}$ satisfies (4.21) we see that the $L_{2}$-norm of $\chi_{A_{1}}(x) f_{j}(x)$ is bounded with a bound that is independent of $x$. Hence we get in the same way as the basic estimate (4.15) is proved in [4] (formula (2.4)) that for $\alpha^{2}<2 \pi$ and $|\lambda| \leqq \lambda_{1}(\alpha)$ [possibly with a smaller bound $\lambda_{1}(\alpha)$ ] we have

$$
\frac{1}{2} \leqq\left\langle e^{-\lambda \tilde{U}_{\Delta_{i}}}\right\rangle_{\hat{\partial} \Delta_{\imath}} \leqq \frac{3}{2} .
$$

Thus we have

Lemma 4.8. Let $k$ be a fixed integer and

$$
\varphi_{i} \in[0,2 \pi), \quad f_{i} \in C(\Lambda) \quad \text { with } \quad\left|f_{i}(x)\right| \leqq 2 e^{\alpha^{2} / 2 K_{i} \Lambda}(x, x), \quad i=1, \ldots, k .
$$


Then for $\alpha^{2}<2 \pi$ and $|\lambda| \leqq \lambda_{1}(\alpha)$ there is a constant $K_{4}$ independent of $\lambda, A$ and $X$ such that

$$
\left\langle e^{-\lambda \tilde{U}_{A}}\right\rangle_{0}^{-1}\left\langle e^{-\lambda \tilde{U}_{A}-X}\right\rangle_{C(\partial X)} \leqq e^{K_{4}|X|}
$$

where

$$
\tilde{U}_{A}=U_{A}+\sum_{i=1}^{k} c_{\varphi_{t}}^{\Lambda}\left(f_{i}\right)
$$

This was the strengthened version of Lemma 4.3 we needed. The following is the strengthened version of Lemma 4.4:

Lemma 4.9. Let the assumptions be as in Lemma 4.8, then there is a constant $K_{5}$ and a norm $|w|$ on test functions continuous on $S\left(\mathbb{R}^{2 N}\right)$ such that for any $K>0$, for any $\Lambda$ and any $\lambda$ with $|\lambda|<\lambda_{1}(\alpha)$ we have that

$$
\left|\left\langle\int \partial^{\Gamma} \int \prod_{i=1}^{N} c_{\theta_{i}}\left(x_{i}\right) e^{-\lambda \tilde{U}_{A}(\xi)} d \mu_{C(s(\Gamma))}(\xi) d s(\Gamma), w\right\rangle\right| \leqq e^{-K|\Gamma|+K_{5}|\Lambda|}|w| .
$$

Moreover if $w$ is of compact support in $X$ then we may take $|w|=C_{N, X}\|w\|_{\infty}$.

Proof. The only change in the proof of Proposition 2.2 of [4] we have to make in order to prove this lemma is in the Lemma 2.3 of [4]. Instead of that lemma we need to establish the following estimate directly

$$
\left\langle e^{-\lambda \tilde{U}_{\Lambda}}\right\rangle_{C(s(\Gamma))} \leqq e^{K_{0}|A|}
$$

where $K_{0}$ is a constant which only depends on $|\lambda|$ and $\alpha$.

However by Lemma 4.7 we have

$$
\left|\left\langle e^{-\lambda \tilde{U}_{\Lambda}}\right\rangle_{C(s(\Gamma))}\right| \leqq\left\langle e^{-\lambda \tilde{U}_{\lambda}}\right\rangle_{0}
$$

If follows from Theorem 3.4 of [22] and its proof that if $\alpha^{2}<2 \pi$ and $f_{i} \in L_{2}\left(\mathbb{R}^{2}\right)$ then

$$
\left\langle e^{-\lambda \tilde{U}_{A}}\right\rangle_{0} \leqq A e^{B \lambda^{2}\left(|\Lambda|+\sum_{i=1}^{k}\left\|f_{i}\right\|_{2}^{2}\right)}
$$

where $A$ and $B$ are constants independent of $\lambda$ and $A$. By the uniform bound (4.21) we get that if $|\partial \Lambda| \leqq|\Lambda|$ where $|\partial \Lambda|$ is the length of $\partial \Lambda$ and $|\Lambda|$ is the area of $\Lambda$ then there is a constant $C$ such that

$$
\left\langle e^{-\lambda \tilde{U}_{A}}\right\rangle_{0} \leqq A e^{C \lambda^{2}|\Lambda|}
$$

This proves (4.24) and now the proof of Lemma 4.9 follows from the proof of Proposition 2.2 of [4].

Let now $\tilde{S}_{\Lambda}^{\lambda}\left(w,\{\theta\}_{N}\right)$ and $\tilde{T}^{\lambda}\left(\{x\}_{N},\{\theta\}_{N}, \Lambda, X, \Gamma\right)$ be defined as $S_{\Lambda}^{\lambda}$ and $T^{\lambda}$ respectively, but with $\tilde{U}_{\Lambda}=U_{\Lambda}+\sum_{i=1}^{k} c_{\varphi_{i}}^{\Lambda}\left(f_{i}\right)$ instead of $U_{\Lambda}$. 
Lemma 4.10. Let the assumptions be as in Lemma 4.8 and let us in addition assume that $|\partial \Lambda| \leqq|\Lambda|$. Then for any $K>0$ given, there is a constant $\mu_{K}$ depending on $K$ such that for $\left|\lambda_{1}\right|<\lambda_{1}(\alpha)$ and for $m_{0}>\mu_{K}$ there is an $S$-norm $|\cdot|$ such that

$$
\sum_{\substack{X, \Gamma \\|X| \geqq D}}\left|\int \tilde{T}^{\lambda}\left(\{x\}_{N},\{\theta\}_{N}, \Lambda, X, \Gamma\right) w\left(\{x\}_{N}\right) d\{x\}_{N}\right| \leqq|w| e^{-K(D-N)}
$$

where $\tilde{T}^{\lambda}$ is defined as $T^{\lambda}$ in (4.16) but with $\tilde{U}_{\Lambda}$ instead of $U_{\Lambda}$. Moreover if $w$ has a fixed compact support inside $X$ then we may take $|w|=C_{N, X}\|w\|_{\infty}$.

Proof. The inequality of the lemma follows by combining the combinatorial estimate (4.18) with Lemma 4.2, Lemma 4.8, and Lemma 4.9.

Now if supp $w=X_{0}$ and $X_{0}$ is a product of unit squares in $\mathbb{R}^{2}$ we set $C_{N, X_{0}} \equiv C_{N}$ since $C_{N, X_{0}}$ obviously is independent of $X_{0}$ as long as $X_{0}$ is a product of unit squares. Now if $d(X)$ is the diameter of $X \subset \mathbb{R}^{2 N}$ then $X$ is contained in the union of $d(X)^{2 N}$ unit squares. From this it follows that the constant $C_{N, X_{0}}$ of Lemma 4.10 satisfies the following inequality

$$
C_{N, X} \leqq C_{N} \cdot d(X)^{2 N}
$$

From Lemma 4.10 and (4.25) we now have the following theorem

Theorem 4.2. Let $\alpha^{2}<2 \pi, k$ a fixed integer and

$$
\varphi_{i} \in[0,2 \pi), \quad f_{i}(x) \in C(\operatorname{Int} \Lambda) \quad \text { and } \quad\left|f_{i}(x)\right| \leqq 2 e^{\alpha^{2} / 2 K_{\hat{\imath} \Lambda}(x, x)}
$$

and set

$$
\tilde{U}_{\Lambda}=U_{A}+\sum_{i=1}^{k} c_{\varphi_{i}}^{\Lambda}\left(f_{i}\right)
$$

Then for $|\lambda| \leqq \lambda_{1}(\alpha)$ and $m_{0} \geqq \mu_{K}$ and $\|w\|_{\infty} \leqq C d(\operatorname{supp} w)^{-2 N}$ we have that the cluster expansion

$$
\begin{aligned}
& \tilde{S}_{\Lambda}^{\lambda}\left(w,\{\theta\}_{N}\right)=\sum_{X, \Gamma}\left\langle e^{-\lambda \tilde{U}_{\Lambda}}\right\rangle_{0}^{-1}\left\langle e^{-\lambda \tilde{U}_{\Lambda}-X}\right\rangle_{C(\partial X)} \\
& \int_{0}^{1} \ldots \int_{0}^{1} \prod_{b \in \Gamma} d s_{b} \frac{\partial}{\partial s_{b}}\left\langle\left(\int \prod_{j=1}^{N} c_{\theta_{J}}\left(x_{j}\right) d x_{j} w\{x\}_{N}\right) e^{-\lambda \tilde{U}_{\Lambda \cap X}}\right\rangle_{C(s(\Gamma))}
\end{aligned}
$$

converges absolutely and uniformly in $\lambda, \Lambda, m_{0}, \varphi_{i}, f_{i}$ and $w$ for $|\lambda| \leqq \lambda_{1}(\alpha), m_{0} \geqq \mu_{K}$, $\left|f_{i}(x)\right| \leqq 2 e^{\alpha^{2} / 2 K_{\partial \Lambda}(x, x)},|\partial \Lambda| \leqq|\Lambda|$ and $(d(\operatorname{supp} w))^{2 N}\|w\|_{\infty} \leqq C$, where $d(\operatorname{supp} w)$ is the diameter of the support of $w$.

Let now

$$
\tilde{S}_{\Lambda, \partial \Lambda}^{\lambda}\left(\{x, \theta\}_{N}\right)=\left\langle e^{-\lambda \tilde{U}_{\Lambda}}\right\rangle_{C(\partial \Lambda)}^{-1}\left\langle\prod_{j=1}^{N} c_{\theta_{j}}\left(x_{j}\right) e^{-\lambda \tilde{U}_{\Lambda}}\right\rangle_{C(\partial \Lambda)},
$$


where $C(\partial \Lambda)=\left(-\Delta_{\hat{\partial} \Lambda}+m_{0}^{2}\right)^{-1}, \Delta_{\partial \Lambda}$ is the Laplacian with Dirichlet boundary conditions on $\partial \Lambda$ and

$$
\tilde{U}_{\Lambda}=U_{\Lambda}+\sum_{i=1}^{k} c_{\varphi_{i}}^{\Lambda}\left(f_{i}\right)
$$

Let \langle\rangle$_{C(s(\Gamma), \hat{c} \Lambda)}$ be constructed from \langle\rangle$_{C(\hat{\partial} \Lambda)}$ in the same way as \langle\rangle$_{C(s(\Gamma))}$ was constructed from \langle\rangle$_{0}$. The following theorem then follows from the proof of Theorem 4.2.

Theorem 4.3. Let the assumptions be as in Theorem 4.2. Then for $|\lambda| \leqq \lambda_{1}(\alpha)$ and $m_{0} \geqq \mu_{K}$ and $d(\operatorname{supp} w)^{2 N}\|w\|_{\infty} \leqq C$ we have that the cluster expansion

$$
\begin{aligned}
& \tilde{S}_{\Lambda, \hat{o} \Lambda}^{\lambda}\left(w,\{\theta\}_{N}\right)=\sum_{X, \Gamma}\left\langle e^{-\lambda \tilde{U}_{\Lambda}}\right\rangle_{C(\hat{\partial} \Lambda)}^{-1}\left\langle e^{-\lambda \tilde{U}_{\Lambda}-X}\right\rangle_{C(\hat{\partial} X \cup \hat{\partial} \Lambda)} \\
& \int_{0}^{1} \ldots \int_{0}^{1} \prod_{b \in \Gamma} d s_{b} \frac{\partial}{\partial s_{b}}\left\langle\left(\int \prod_{j=1}^{N} c_{\theta_{j}}\left(x_{j}\right) d x_{j} w\{x\}_{N}\right) e^{-\lambda \tilde{U}_{A \cap X}}\right\rangle_{C(S(\Gamma), \hat{\partial} \Lambda)}
\end{aligned}
$$

converges absolutely and uniformly in $\lambda, \Lambda, m_{0}, \varphi_{i}, f_{i}$ and $w$ for $|\lambda| \leqq \lambda_{1}(\alpha), m_{0} \geqq \mu_{K}$, $\left|f_{i}(x)\right| \leqq 2 e^{\alpha^{2} / 2 K_{i A}(x, x)}$ and $|\partial \Lambda|<|\Lambda|,(d(\operatorname{supp} w))^{2 N}\|w\|_{\infty} \leqq C$ where $d(\operatorname{supp} w)$ is the diameter of the support of $w$.

Now exactly the same way as the convergence of the cluster expansion (4.14) implies the exponential cluster property for $S_{\Lambda}^{\lambda}(w,\{\theta\}$ ) (see [4], p. 901 and also [2], Sect. 4) we get that Theorem 4.3 implies the following lemma

Lemma 4.11. Let the assumptions be as in Theorem 4.2. Let $w\left(\{x\}_{N}\right)$ and $w^{\prime}\left(\{x\}_{N^{\prime}}\right)$ have compact support $X$ and $X^{\prime}$ respectively and let $d(X)$ and $d\left(X^{\prime}\right)$ be the corresponding diameters and $d\left(X, X^{\prime}\right)$ the distance between $X$ and $X^{\prime}$. Then there are positive constants $a, \lambda_{1}, \mu_{1}$ and $C_{N, N^{\prime}}$ such that

$$
\begin{aligned}
& \left|\tilde{S}_{\Lambda, \partial \Lambda}^{\lambda}\left(w \otimes w^{\prime},\{\theta\}_{N} \times\{\theta\}_{N^{\prime}}\right)-\tilde{S}_{\Lambda, \partial \Lambda}^{\lambda}\left(w,\{\theta\}_{N}\right) \cdot \tilde{S}_{\Lambda, \partial \Lambda}^{\lambda}\left(w^{\prime},\{\theta\}_{N^{\prime}}\right)\right| \\
& \quad \leqq C_{N, N^{\prime}} e^{-\operatorname{ad}\left(X, X^{\prime}\right)} \cdot d(X)^{2 N} \cdot d\left(X^{\prime}\right)^{2 N^{\prime}}\|w\|_{\infty}\left\|w^{\prime}\right\|_{\infty}
\end{aligned}
$$

for $|\lambda| \leqq \lambda_{1}, m_{0} \geqq \mu_{1},|\partial \Lambda| \leqq|\Lambda|$ and $\left|f_{i}(x)\right| \leqq 2 e^{\alpha^{2} / 2 K_{\bar{\tau} \Lambda}(x, x)}, i=1, \ldots, k$.

$$
\begin{aligned}
& \text { Let now } U_{\Lambda}^{\alpha}=U_{A}+\alpha \sum_{i=1}^{k} c_{\varphi_{l}}^{\Lambda}\left(f_{i}\right) \text {, and set } \\
& S_{\Lambda, \partial \Lambda}^{\lambda, \alpha}\left(\{x, \theta\}_{N}\right)=\left\langle e^{-\lambda U_{A}^{\alpha}}\right\rangle_{C(\partial \Lambda)}^{-1}\left\langle\prod_{j=1}^{N} c_{\theta_{j}}\left(x_{j}\right) e^{-\lambda U_{A}^{\alpha}}\right\rangle_{C(\hat{\partial} A)} .
\end{aligned}
$$

Then

$$
\begin{aligned}
& \frac{d}{d \alpha} S_{\Lambda, \partial \Lambda}^{\lambda, \alpha}\left(\{x, \theta\}_{N}\right)=-\lambda \sum_{i=1}^{k}\left(S_{\Lambda, \partial \Lambda}^{\lambda, \alpha}\left(\{x, \theta\}_{N} \otimes\left\{\chi_{\Lambda} f_{i}, \varphi_{i}\right\}\right)\right. \\
& \left.\quad-S_{\Lambda, \hat{o} \Lambda}^{\lambda, \alpha}\left(\{x, \theta\}_{N}\right) \cdot S_{\Lambda, \hat{o} \Lambda}^{\lambda, \alpha}\left(\chi_{\Lambda} f_{i}, \varphi_{i}\right)\right)
\end{aligned}
$$


where $\chi_{A}$ is the characteristic function for the set $\Lambda$. By integrating (4.28) over $\alpha$ from zero to one and using Lemma 4.11 we get:

Lemma 4.12. With the notations of Lemma 4.11 there is a constant $C_{N}$ depending only on $N$ and $k$ such that

$$
\begin{aligned}
& \left.\mid \tilde{S}_{\Lambda, \partial \Lambda}^{\lambda}\left(w,\{\theta\}_{N}\right\}\right)-S_{\Lambda, \hat{o} \Lambda}^{\lambda}\left(w,\{\theta\}_{N}\right) \mid \\
& \quad \leqq C_{N}\left(e^{-\operatorname{ad}(X, \Lambda-Y)} d(\Lambda)^{2}+\varepsilon d(Y)^{2}\right) d(X)^{2 N}\|w\|_{\infty}
\end{aligned}
$$

if $\left|f_{i}(x)\right| \leqq \varepsilon$ for $x \in Y$ and $X_{\alpha^{2}}=\operatorname{supp} w$, with $w=w\left(x_{1}, \ldots, x_{N}\right) ;$ for $|\lambda| \leqq \lambda_{1}$, $m_{0} \geqq \mu_{1},|\partial \Lambda| \leqq|\Lambda|$ and $\left|f_{i}(x)\right| \leqq 2 e^{\frac{\alpha^{2}}{2} K_{i \Lambda}(x, x)}, i=1, \ldots, k$.

Let now $B_{n}=\left\{x \in \mathbb{R}^{2} ;|x| \leqq n\right\}$ and $C_{n}=\partial B_{n}$. Let $f_{i, n}, i=1, \ldots, k$ be in $C\left(\operatorname{Int} B_{n}\right)$ with $\left|f_{i, n}(x)\right| \leqq 2 e^{\frac{\alpha^{2}}{2} K_{i \wedge}(x, x)}$ such that for $m \leqq n-1$ we have

$$
\sup _{x \in B_{m}}\left|f_{i, n}(x)\right| \leqq b e^{-\frac{x^{\prime}}{2}(n-m)}
$$

where $b$ and $\alpha^{\prime}$ are positive constants independent of $n$ and $m$. From Lemma 4.12 and (4.29) we then get with $m=\frac{n}{2}, Y=B_{n / 2}$ and $\varepsilon=b e^{-\frac{\alpha^{\prime}}{4} n}$

$$
\begin{aligned}
& \left|\tilde{S}_{B_{n}, \hat{c} B_{n}}^{\lambda, n}\left(w,\{\theta\}_{N}\right)-S_{B_{n}, \hat{c} B_{n}}^{\lambda}\left(w,\{\theta\}_{N}\right)\right| \\
& \quad \leqq C_{N}\left(e^{-a\left(\frac{n}{2}-d(X)\right)} \cdot n^{2}+b e^{-\frac{\alpha^{\prime}}{4} n}\left(\frac{n}{2}\right)^{2}\right) d(X)^{2 N}\|w\|_{\infty} .
\end{aligned}
$$

Now the convergence of the cluster expansion for $S_{\Lambda, c \Lambda}^{\lambda}\left(w,\{\theta\}_{N}\right)$ uniformly in $\Lambda$ is of course a consequence of Theorem 4.3. From this it follows in the same way as in Theorem 4.1 that

$$
S_{\Lambda, \hat{i} \Lambda}^{\lambda}\left(w,\{\theta\}_{N}\right) \rightarrow S^{\lambda}\left(w,\{\theta\}_{N}\right)
$$

as $\Lambda \nearrow R^{2}$. From (4.30) we then get the main theorem of this section.

Theorem 4.4. Let $\alpha^{2}<2 \pi, k$ a fixed integer and $\varphi_{i} \in[0,2 \pi)$. Let $f_{i, n} \in C\left(B_{n}\right)$, $B_{n}=\left\{x \in R^{2} ;|x|<n\right\}$ such that $\left|f_{i, n}(x)\right| \leqq 2 e^{\frac{\alpha^{2}}{2} K_{i B_{n}}(x, x)}$ and for $m \leqq n-1$

$$
\sup _{x \in B_{m}}\left|f_{i, n}(x)\right| \leqq b e^{-\frac{x^{\prime}}{2}(n-m)}
$$

where $b$ and $\alpha^{\prime}$ are positive constants independent of $n$ and $m$. Let

$$
\tilde{U}_{B_{n}}^{n}=U_{B_{n}}+\sum_{i=1}^{k} c_{\varphi_{i}}\left(f_{i, n}\right)
$$

and

$$
\tilde{S}_{B_{n}, \hat{C} B_{n}}^{\lambda, n}\left(w,\{\theta\}_{N}\right)=\left\langle e^{-\lambda \tilde{U}_{B_{n}}^{n}}\right\rangle_{C\left(\hat{\partial} B_{n}\right)}^{-1} \int\left\langle\prod_{j=1}^{N} c_{\theta_{J}}\left(x_{j}\right) e^{-\lambda \tilde{U}_{B_{n}}^{n}}\right\rangle_{C\left(\hat{c} B_{n}\right)} w\left(\{x\}_{N}\right) d\{x\}_{N} .
$$

Then

$$
\tilde{S}_{B_{n}, \partial B_{n}}^{\lambda, n}\left(w,\{\theta\}_{N}\right) \rightarrow S^{\lambda}\left(w,\{\theta\}_{N}\right)
$$


as $n \rightarrow \infty$, whenever $w=w\left(x_{1}, \ldots, x_{N}\right)$ is bounded measurable and of bounded support.

\section{Uniqueness for the Trigonometric Interaction}

Let $E_{0}$ respectively $E_{\lambda}$ be the expectations with respect to the measure $\mu_{0}$ given by the free Euclidean field of mass $m$ and respectively a measure $\mu_{\lambda} \in G^{\lambda U}$ such that $\mu_{\lambda}$ is a regular random field. Let now $B_{n}=\left\{x \in \mathbb{R}^{2} ;|x|<n\right\}$ and $C_{n}=\partial B_{n}$. From (3.11) we have

$$
E_{0}(f \mid C)(\eta)=E_{0}^{C}\left(f\left(\xi+\psi_{\eta}^{C}\right)\right)
$$

where $E_{0}^{C}$ is the expectation with respect to the Gaussian measure of mean zero and covariance $\left(-\Delta_{C}+m_{0}^{2}\right)^{-1}, \Delta_{C}$ being the Laplacian with Dirichlet boundary conditions on $C$. Then

$$
E_{0}(f \mid C)(\eta)=\int_{S^{\prime}\left(\mathbb{R}^{2}\right)} f(\xi) d \mu_{0}^{C}(\xi \mid \eta)
$$

where $\mu_{0}^{C}(\xi \mid \eta)$ is the Gaussian measure with mean $\psi_{\eta}^{C}(x)$ and covariance $\left(-\Delta_{C}+m_{0}^{2}\right)^{-1}$. Since $\mu_{\lambda} \in G^{\lambda U}$, where $\Lambda \rightarrow U_{A}$ is the additive functional given by (4.1), we have by (3.16) that

$$
E_{\lambda}(f \mid C)(\eta)=\int_{S^{\prime}\left(\mathbb{R}^{2}\right)} f(\xi) d \mu_{\lambda}^{C}(\xi \mid \eta)
$$

with

$$
d \mu_{\lambda}^{C}(\xi \mid \eta)=\left(E_{0}\left(e^{-\lambda U_{B}} \mid C\right)(\eta)\right)^{-1} e^{-\lambda U_{B}} d \mu_{0}^{C}(\xi \mid \eta)
$$

where $B$ is a bounded domain and $\partial B=C$.

Hence by (5.1)

$$
\begin{aligned}
E_{\lambda}(f \mid C)(\eta) & =\left(E_{0}\left(e^{-\lambda U_{B}} \mid C\right)(\eta)\right)^{-1} E_{0}\left(f e^{-\lambda U_{B}} \mid C\right)(\eta) \\
& =E_{0}^{C}\left(e^{-\lambda U_{B}\left(\xi+\psi_{\eta}^{c}\right)}\right)^{-1} E_{0}^{C}\left(f\left(\xi+\psi_{\eta}^{C}\right) e^{-\lambda U_{B}\left(\xi+\psi_{\eta}^{c}\right)}\right) .
\end{aligned}
$$

Let now $f(\xi)=e^{i\langle\varphi, \xi\rangle}$ for some $\varphi \in S\left(R^{2}\right)$, and set $U_{B}\left(\xi+\psi_{\eta}^{C}\right)=U_{B}^{\eta}$, then we get

$$
E_{\lambda}\left(e^{i\langle\varphi, \xi\rangle} \mid C\right)(\eta)=e^{i\left\langle\varphi, \psi_{\eta}^{c}\right\rangle} E_{0}^{C}\left(e^{-\lambda U_{B}^{\eta}}\right)^{-1} E_{0}^{C}\left(e^{i\langle\varphi, \xi\rangle} e^{-\lambda U_{B}^{\eta}}\right) .
$$

We shall prove that $E_{\lambda}\left(e^{i\langle\varphi, \xi\rangle} \mid C_{n}\right)(\eta) \rightarrow E_{\lambda}\left(e^{i\langle\varphi, \xi\rangle}\right) \mu_{\lambda}$-almost surely as $n \rightarrow \infty$. From Theorem 2.1 we have that if $\Lambda_{n} \subset B_{n}$ such that $d\left(A_{n}, C_{n}\right) \rightarrow \infty$ then for any $\alpha^{\prime}<m_{0}$ there is a subsequence $n^{\prime}$ such that

$$
e^{\alpha^{\prime} / 2 d\left(\Lambda_{n^{\prime}}, C_{n^{\prime}}\right)} \sup _{x \in \Lambda_{n^{\prime}}}\left|\psi_{\eta}^{C_{n^{\prime}}}(x)\right| \rightarrow 0
$$

for $\mu_{\lambda}$-almost every $\zeta$, because $\mu_{\lambda}$ is a regular Markov field. (5.7) implies that

$$
e^{i\left\langle\varphi, \psi_{\eta}^{C_{n}}\right\rangle} \rightarrow 1
$$

for $\mu_{\lambda}$-almost all $\eta$.

From the fact that $\xi(x)$ and $\psi_{\xi}^{C}(x)$ are independent Gaussian fields with covariance $G_{C}(x, y)$ and $K_{C}(x, y)$ respectively [see (2.11) and (3.10)] we have by 
using the trigonometric formula for cosinus

$$
\begin{aligned}
U_{B}^{\eta}(\xi)= & \int_{B}: \cos \left(\alpha \xi(x)+\theta+\alpha \psi_{\eta}^{C}(x)\right): d x \\
= & \int_{B}: \cos (\alpha \xi(x)+\theta):: \cos \alpha \psi_{\eta}^{C}(x): d x \\
& -\int: \sin (\alpha \xi(x)+\theta):: \sin \alpha \psi_{\eta}^{C}(x): d x .
\end{aligned}
$$

Hence in the notation of (4.4)

$$
U_{B}^{\eta}(\xi)=U_{B}(\xi)+c_{\theta}\left(f_{\eta}\right)+c_{\theta-(\pi / 2)}\left(g_{\eta}\right)
$$

with

$$
f_{\eta}=1-: \cos \alpha y_{\eta}^{C}(x):=1-e^{\alpha^{2 / 2} K_{C}(x, x)} \cos \alpha \psi_{\eta}^{C}(x)
$$

and

$$
g_{\eta}(x)=-: \sin \alpha \psi_{\eta}^{C}(x):=-e^{x^{2} / 2 K_{C}(x, x)} \sin \alpha \psi_{\eta}^{C}(x) .
$$

From (5.7) and Theorem 4.4 we get that

$$
\begin{aligned}
& E_{0}^{C_{n^{\prime}}}\left(e^{\left.-\lambda U_{B n^{\prime}}^{\eta}\right)^{-1}} E_{0}^{C_{n^{\prime}}}\left(\int \prod_{j=1}^{N}\left(c_{\theta_{j}}\left(x_{j}\right) d x_{j}\right) w\left(x_{1}, \ldots, x_{N}\right) e^{-\lambda U_{B n^{\prime}}^{\eta}}\right)\right. \\
& \quad \rightarrow E_{\lambda}\left(\int \prod_{j=1}^{N}\left(c_{\theta_{j}}\left(x_{j}\right) d x_{j}\right) w\left(x_{1}, \ldots, x_{N}\right)\right)
\end{aligned}
$$

for some subsequence $n^{\prime}$ of natural numbers.

Since $\mu_{\lambda}$ is locally equivalent to $\mu_{0}$ and $c_{\theta}(x)$ generates the $\sigma$-algebra of $\mu_{0}$-measurable sets the convergence (5.12) implies that

$$
E_{0}^{C_{n^{\prime}}}\left(e^{-\lambda U_{B^{\prime}}{ }^{\eta}}\right)^{-1} E_{0}^{C_{n^{\prime}}}\left(e^{i\langle\varphi, \xi\rangle} e^{-\lambda U_{B^{\prime}}{ }^{\prime}}\right) \rightarrow E_{\lambda}\left(e^{i\langle\varphi, \xi\rangle}\right)
$$

for $\varphi \in S\left(\mathbb{R}^{2}\right)$ with compact support, for $\mu_{\lambda}$-almost all $\eta \in S^{\prime}\left(\mathbb{R}^{2}\right)$.

Since the functions of compact support are dense in $S\left(\mathbb{R}^{2}\right)$ and $\mu_{0}$ and $\mu_{i}$ are measures on $S^{\prime}\left(\mathbb{R}^{2}\right)$ we see that the convergence (5.13) holds for any $\varphi \in S\left(\mathbb{R}^{2}\right)$. Now (5.13) together with (5.8) give that for any $\varphi \in S\left(\mathbb{R}^{2}\right)$

$$
E_{\lambda}\left(e^{i\langle\varphi, \xi\rangle} \mid C_{n^{\prime}}\right)(\eta) \rightarrow E_{\lambda}\left(e^{i\langle\varphi \cdot \xi\rangle}\right)
$$

for $\mu_{\lambda}$-almost all $\eta \in S^{\prime}\left(\mathbb{R}^{2}\right)$, where $n^{\prime}$ is some subsequence of the sequence $n$ of natural numbers. From (3.25) we have that

$$
E_{\lambda}\left(\cdot \mid \mathbb{R}^{2}-\Lambda\right) \rightarrow E_{\lambda}\left(\cdot \mid B_{\infty}\right)
$$

monotonously as $\Lambda \nearrow \mathbb{R}^{2}$ in the ordered family of bounded open sets and the convergence is monotone and strong in the sense of operators on $L_{2}\left(d \mu_{\lambda}\right)$. If $\varphi$ has bounded support we have that

$$
E_{\lambda}\left(e^{i\langle\varphi, \xi\rangle} \mid C_{n}\right)=E_{\lambda}\left(e^{i\langle\varphi, \xi\rangle} \mid \mathbb{R}^{2}-B_{n}\right)
$$

as soon as $\operatorname{supp} \varphi \subset B_{n}$. Therefore (5.15) together with (5.14) implies that

$$
E_{\lambda}\left(e^{i\langle\varphi, \xi\rangle} \mid \mathbb{R}^{2}-\Lambda\right) \rightarrow E_{\lambda}\left(e^{i\langle\varphi, \xi\rangle}\right)
$$

as $\Lambda$ converges to $\mathbb{R}^{2}$ in the ordered family of bounded open subsets of $\mathbb{R}^{2}$. 
By Theorem 3.2 we have proved that $\mu_{\lambda}$ is an extreme phase. Now the only condition on $\mu_{\lambda}$ was that $\mu_{\lambda} \in G^{\lambda U}$, i.e. that $\mu_{\lambda}$ is a Gibbs measure for the trigonometric interaction (4.1) and that $\mu_{\lambda}$ is a regular random field. If there are two regular random fields $\mu_{\lambda}^{1}$ and $\mu_{\lambda}^{2}$ in $G^{\lambda U}$ then $\mu_{\lambda}^{3}=\frac{1}{2} \mu_{\lambda}^{1}+\frac{1}{2} \mu_{\lambda}^{2}$ is also a regular random field in $G^{\lambda U}$ and since $\mu_{\lambda}^{1}$ and $\mu_{\lambda}^{2}$ both are extreme phases $\mu_{\lambda}^{3}$ is not an extreme phase.

Hence we have the following theorem.

Theorem 5.1. Let $\alpha^{2}<2 \pi$ and $m_{0}>0$, then there is $a \lambda_{1}>0$ depending only on $\alpha$ and $m_{0}$ such that for $-\lambda_{1} \leqq \lambda_{1} \leqq \lambda_{1}$ there is one and only one measure $\mu_{\lambda}$ corresponding to a regular random field $\xi(x)$ with $\mu_{\lambda} \in G^{\lambda U}$, where $G^{\lambda U}$ is the set of all Gibbs measures corresponding to the additive functional

$$
\lambda U_{\Lambda}(\xi)=\lambda \int_{A}: \cos (\alpha \xi(x)+\theta): d x .
$$

We recall that $\xi(x)$ is said to be a regular random field if the corresponding probability measure is supported by $S^{\prime}\left(\mathbb{R}^{2}\right)$ and there is a constant $c$ such that

$$
E\left(|\langle\varphi, \xi\rangle|^{2}\right)<c \int G_{m}(x-y) \varphi(x) \varphi(y) d x d y
$$

for any $\varphi \in S(\mathbb{R})$, for some fixed positive $m$.

For $-\lambda_{1} \leqq \lambda \leqq \lambda_{1}$ we have especially that $\mu_{\lambda} \in G^{\lambda U}$ is an extreme phase.

From the proof of Theorem 5.1 we also have

Theorem 5.2. Let $\alpha^{2}<2 \pi$ and $m_{0}>0$ then there is $a \lambda_{1}>0$ depending only on $\alpha$ and $m_{0}$ such that for $-\lambda_{1}<\lambda<\lambda_{1}$ we have that if $\eta \in S^{\prime}\left(\mathbb{R}^{2}\right)$ such that $\psi_{\eta}^{\partial \Lambda}(x) \rightarrow 0$ locally uniformly as $\Lambda \uparrow \mathbb{R}^{2}$ then

$$
d \mu_{\Lambda}^{\eta}(\xi)=\frac{e^{-\lambda U_{\Lambda}(\xi)}}{E_{0}\left(e^{-\lambda U_{\Lambda}} \mid \partial \Lambda\right)(\eta)} d \mu_{0}^{i \Lambda}(\xi \mid \eta)
$$

converge weakly to the unique limit $\mu$ given in Theorem 5.1, where $U_{A}$ is the trigonometric interaction of Theorem 5.1 and $\mu_{0}^{\partial \Lambda}(\xi \mid \eta)$ is the free Euclidean field with boundary condition $\xi=\eta$ on $\partial \Lambda$ defined in Sect. 2 by

$$
\int f(\xi) d \mu_{0}^{\partial \Lambda}(\xi \mid \eta)=\int f\left(\xi+\psi_{\eta}^{\partial \Lambda}\right) d \mu_{0}^{\partial \Lambda}(\xi)
$$

where $\mu_{0}^{\hat{c \Lambda}}$ is the free field with Dirichlet boundary conditions on $\partial \Lambda$.

\section{The Global Markov Property for the Trigonometric Interactions}

Let now $C_{0}$ be an unbounded connected piecewise $C^{1}$-curve such that $\mathbb{R}^{2}-C_{0}$ consists of two components $\Omega_{+}$and $\Omega_{-}$. Let $B_{n}=\left\{x \in \mathbb{R}^{2} ;|x|<n\right\}, n \in N$ and $C_{n}=\partial B_{n}-C_{0}$, where $\partial B_{n}=\{|x|=n\}$ is the boundary of $B_{n}$. We also assume that, for any $n \in N, \partial B_{n} \cap C_{0}$ consists of at most a finite number of points. From (5.3) we then have that if $f$ is $B_{B_{n}}$-measurable then

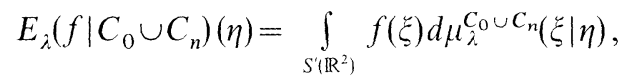


with

$$
\mathrm{d} \mu_{\lambda}^{C_{0} \cup C_{n}}(\xi \mid \eta)=\left[E_{0}\left(e^{-\lambda U} B_{n} \mid C_{0} \cup C_{n}\right)(\eta)\right]^{-1} e^{-\lambda U_{B_{n}}} d \mu_{0}^{C_{0} \cup C_{n}}(\xi \mid \eta) .
$$

Thus by (5.5), with $U_{n}=U_{B_{n}}$,

$$
\begin{gathered}
E_{\lambda}\left(f \mid C_{0} \cup C_{n}\right)(\eta)=\left[E _ { 0 } ^ { C _ { 0 } \cup C _ { n } } \left(e^{\left.-\lambda U_{n}\left(\xi+\psi_{\eta}^{\left.C_{0} \cup C_{n}\right)}\right)\right]^{-1}}\right.\right. \\
\cdot E_{0}^{C_{0} \cup C_{n}}\left(f\left(\xi+\psi_{\eta}^{C_{0} \cup C_{n}}\right) e^{-\lambda U_{n}\left(\xi+\psi_{\eta}^{\left.C_{0} \cup C_{n}\right)}\right) .}\right.
\end{gathered}
$$

Set now $\psi_{\eta}^{n}(x)=\psi_{\eta}^{C_{0} \cup C_{n}}(x)-\psi_{\eta}^{C_{0}}(x), f(\xi)=e^{i\langle\varphi, \xi\rangle}$, then (6.3) takes the form

$$
\begin{gathered}
E_{\lambda}\left(e^{i\langle\varphi . \xi\rangle} \mid C_{0} \cup C_{n}\right)(\eta)=e^{i\left\langle\varphi, \psi_{\eta}^{\left.C_{0}\right\rangle} e^{i\left\langle\varphi, \psi_{\eta}^{n}\right\rangle}\right.} \\
\cdot\left[E_{0}^{C_{0} \cup C_{n}}\left(e^{-\lambda U_{n}\left(\xi+\psi_{\eta} C_{0}+\psi_{\eta}^{n}\right)}\right)\right]^{-1} \\
\cdot E_{0}^{C_{0} \cup C_{n}}\left(e^{i\langle\varphi . \xi\rangle} e^{-\lambda U_{n}\left(\xi+\psi_{\eta}^{\left.C_{0}+\psi_{\eta}^{n}\right)}\right) .}\right.
\end{gathered}
$$

From Theorem 2.1 we have that if $\Lambda_{n} \subset B_{n}$ such that $d\left(\Lambda_{n}, C_{n}\right) \rightarrow \infty$ as $n \rightarrow \infty$, then we have for any $\beta<m_{0}$ that there is a subsequence $N^{\prime} \subset N$ such that if $n^{\prime} \in N^{\prime}$ then

$$
e^{\beta / 2 d\left(\Lambda_{n^{\prime}}, C_{n^{\prime}}\right)} \sup _{x \in \Lambda_{n^{\prime}}}\left|\psi_{\eta}^{n^{\prime}}(x)\right| \rightarrow 0
$$

as $n^{\prime} \rightarrow \infty$, for $\mu_{\lambda}$-almost all $\eta$ in $S^{\prime}\left(\mathbb{R}^{2}\right)$. Thus

$$
e^{i\left\langle\varphi, \psi_{\eta}^{n^{\prime}}\right\rangle} \rightarrow 1
$$

for $\mu_{\lambda}$-almost all $\eta$, as $n^{\prime} \rightarrow \infty$.

Let now for $\eta \in S^{\prime}\left(\mathbb{R}^{2}\right)$

$$
\begin{aligned}
\tilde{S}_{C_{0} \cup C_{n}}^{\eta}\left(\{x, \theta\}_{k}\right) \equiv\left[E _ { 0 } ^ { C _ { 0 } \cup C _ { n } } \left(e^{\left.-\lambda U_{n}\left(\xi+\psi_{\eta}^{\left.C_{0}+\psi_{\eta}^{n}\right)}\right)\right]^{-1}}\right.\right. \\
\cdot E_{0}^{C_{0} \cup C_{n}}\left(\left(\prod_{j=1}^{k} c_{\theta_{j}}\left(x_{j}\right)\right) e^{-\lambda U_{n}\left(\xi+\psi_{\eta}^{\left.C_{0}+\psi_{\eta}^{n}\right)}\right.}\right)
\end{aligned}
$$

and

$$
\begin{aligned}
S_{C_{0} \cup C_{n}}^{\eta}\left(\{x, \theta\}_{k}\right) \equiv & {\left[E_{0}^{C_{0} \cup C_{n}}\left(e^{-\lambda U_{n}\left(\xi+\psi \eta^{\left.\gamma_{0}\right)}\right)}\right)\right]^{-1} } \\
& \cdot E_{0}^{C_{0} \cup C_{n}}\left(\left(\prod_{j=1}^{k} c_{\theta_{j}}\left(x_{j}\right)\right) e^{-\lambda U_{n}\left(\xi+\psi_{\eta}{ }^{\left.C_{0}\right)}\right.}\right) .
\end{aligned}
$$

We remark now that one has

$$
U_{n}\left(\xi+\psi_{\eta}^{C_{0}}+\psi_{\eta}^{n}\right)=U_{n}\left(\xi+\psi_{\eta}^{C_{0}}\right)+c_{\theta}\left(f_{\eta}\right)+c_{\theta-(\pi / 2)}\left(g_{\eta}\right),
$$

with

$$
f_{\eta}(x) \equiv 1-e^{\alpha^{2} / 2 K_{C}(x, x)} \cos \alpha \psi_{\eta}^{C}(x) \quad \text { and } \quad g_{\eta}(x)=-e^{\alpha^{2} / 2 K_{C}(x, x)} \sin \alpha \psi_{\eta}^{C}(x) .
$$

Using (6.8) and (6.9) together with Lemma 4.11 we get, by making the obvious changes in the proof of Lemma 4.12, the following

Lemma 6.1. There exists a constant a independent of $k$ and a constant $C_{k}^{\prime}$ depending only on $k$ such that, for $|\lambda| \leqq \lambda_{1}$ and $m_{0} \geqq \mu_{1}$ :

$$
\begin{aligned}
& \left|\tilde{S}_{C_{0} \cup C_{n}}^{\eta}\left(w,\{\theta\}_{k}\right)-S_{C_{0} \cup C_{n}}^{\eta}\left(w,\{\theta\}_{k}\right)\right| \\
& \quad \leqq C_{k}^{\prime}\left(e^{-a d\left(X, B_{n}-Y\right)} d(\Lambda)^{2}+\varepsilon d(Y)^{2}\right) d(X)^{2 N}\|w\|_{\infty},
\end{aligned}
$$

if $\left|\psi_{\eta}^{n}(x)\right| \leqq \varepsilon$ for all $x \in Y$ and $X=\operatorname{supp} w$, with $w=w\left(x_{1}, \ldots, x_{k}\right)$. 
Take now $Y=B_{n / 2}$ and $\varepsilon=e^{(-(\beta) / 4) n}$, then we get from Lemma 6.1

$$
\begin{aligned}
& \left|\tilde{S}_{C_{0} \cup C_{n}}^{\eta}\left(w,\{\theta\}_{k}\right)-S_{C_{0} \cup C_{n}}^{\eta}\left(w,\{\theta\}_{k}\right)\right| \\
& \leqq C_{k}^{\prime}\left(e^{-a\left(\frac{n}{2}-d(X)\right)} n^{2}+e^{(-(\beta) / 4) n}\left(\frac{n}{2}\right)^{2}\right) d(X)^{2 k}\|w\|_{\infty}
\end{aligned}
$$

whenever

$$
\sup _{x \in B_{n / 2}}\left|\psi_{\eta}^{n}(X)\right| \leqq e^{(-(\beta) / 4) n}
$$

(and $|\lambda| \leqq \lambda_{1}, m_{0} \geqq \mu_{1}$ ). From (6.5) we have that there exists a subsequence $N^{\prime} \subset N$ such that (taking $\Lambda_{n}=B_{n / 2}$ )

$$
e^{\beta / 4 n^{\prime}} \sup _{x \in B_{n^{\prime} / 2}}\left|\psi_{\eta}^{n^{\prime}}(x)\right| \rightarrow 0
$$

as $n^{\prime} \in N^{\prime}, n^{\prime} \rightarrow \infty$, for $\mu_{\lambda}$-almost all $\eta$. But this implies that

$$
\sup _{x \in B_{n^{\prime} / 2}}\left|\psi_{\eta}^{n^{\prime}}(x)\right| \leqq e^{(-(\beta) / 4) n^{\prime}}
$$

for $n^{\prime} \geqq n_{0}(\eta)$, where $n_{0}(\eta)$ is finite for $\mu_{\lambda}$-almost all $\eta$. Hence there is a subsequence $N^{\prime} \subset N$ such that if $n^{\prime} \in N^{\prime}$ and $n^{\prime} \geqq n_{0}(\eta)$ then (6.10) holds. Hence we have the following

Lemma 6.2. Let $w=w\left(x_{1}, \ldots, x_{k}\right)=w\left(\{x\}_{k}\right)$ be in $C\left(\mathbb{R}^{2 k}\right)$ with compact support. Let

$$
\tilde{S}_{C_{0} \cup C_{n}}^{\eta}\left(w,\{\theta\}_{k}\right) \equiv \int_{\mathbb{R}^{2 k}} \tilde{S}_{C_{0} \cup C_{n}}^{\eta}\left(\{x, \theta\}_{k}\right) w\left(\{x\}_{k}\right) d\{x\}_{k},
$$

where $d\{x\}_{k}=\prod_{j=1}^{k} d x_{j}$, and define accordingly $S_{C_{0} \cup C_{n}}^{\eta}\left(w,\{\theta\}_{k}\right)$, with $\tilde{S}_{C_{0} \cup C_{n}}^{\eta}\left(\{x, \theta\}_{k}\right)$ and $S_{C_{0} \cup C_{n}}^{\eta}\left(\{x, \theta\}_{k}\right)$ given by (6.6) resp. (6.7). Then there exists a subsequence $N^{\prime} \subset N$ such that for $n^{\prime} \in N^{\prime}$

$$
\left|\tilde{S}_{C_{0} \cup C_{n^{\prime}}}^{\eta}\left(w,\{\theta\}_{k}\right)-S_{C_{0} \cup C_{n^{\prime}}}^{\eta}\left(w,\{\theta\}_{k}\right)\right| \rightarrow 0
$$

as $n^{\prime} \rightarrow \infty$, whenever $|\lambda| \leqq \lambda_{1}$ and $m_{0} \geqq \mu_{1}$. The subsequence $N^{\prime} \subset N$ does not depend on $k$ or $w$.

From (6.7) we see that $S_{C_{0} \cup C_{n}}^{\eta}$ depends on $\eta$ only through the random field $\psi_{\eta}^{C_{0}}(x)$. From (2.4) we have that $\psi_{\eta}^{C_{0}}(x)$ is $B_{C_{0}}$-measurable, where $B_{C_{0}}$ is the $\sigma$ algebra generated by the functions $\eta \rightarrow\langle\eta, \varrho\rangle$, where $\varrho$ is a measure of finite support, supp $\varrho \subset C_{0}$, and with finite energy. Hence the functions

$$
\eta \rightarrow S_{C_{0} \cup C_{n},}^{\eta}\left(w,\{\theta\}_{k}\right)
$$

are all $B_{C_{0}}$-measurable. On the other hand by (6.6) we have

$$
\tilde{S}_{C_{0} \cup C_{n^{\prime}}}^{\eta}\left(\{x, \theta\}_{k}\right)=E_{\lambda}\left(\left[\prod_{j=1}^{k}\left(c_{\theta_{j}}\left(x_{j}\right)\right)\right]\left(\xi-\psi_{\eta}^{C_{0}}-\psi_{\eta}^{n}\right) \mid C_{0} \cup C_{n^{\prime}}\right)(\eta) .
$$

But

$$
\begin{aligned}
& c_{\theta}(x)\left(\xi-\psi_{\eta}^{C_{0}}-\psi_{\eta}^{n}\right)=\cos \left(\alpha\left(\psi_{\eta}^{C_{0}}(x)-\psi_{\eta}^{n}(x)\right)\right) \cdot c_{\theta}(x)(\xi) \\
& \quad+\sin \left(\alpha\left(\psi_{\eta}^{C_{0}}(x)-\psi_{\eta}^{n}(x)\right)\right) \cdot c_{\theta-\frac{\pi}{2}}(x)(\xi) .
\end{aligned}
$$


Hence $\tilde{S}_{C_{0} \cup C_{n^{\prime}}}^{\eta}\left(\{x, \theta\}_{k}\right)$ may be expressed as a finite sum of terms of the form

$$
\begin{aligned}
& \prod_{j=1}^{l}\left[\cos \left(\alpha\left(\psi_{\eta}^{C_{0}}\left(x_{j}\right)+\psi_{\eta}^{n}\left(x_{j}\right)\right)\right)\right] \prod_{i=1}^{m}\left[\sin \left(\alpha\left(\psi_{\eta}^{C_{0}}\left(y_{i}\right)+\psi_{\eta}^{n}\left(y_{i}\right)\right)\right)\right] \\
& \left.E_{\lambda}\left(\prod_{j=1}^{l} c_{\theta_{j}}\left(x_{j}\right) \prod_{i=1}^{l} c_{\theta_{i}-\frac{\pi}{2}}\left(y_{i}\right)\right) C_{0} \cup C_{n^{\prime}}\right)(\eta) .
\end{aligned}
$$

The second line in (6.17) is by the local Markov property of $\mu_{\lambda}$ equal to

$$
E_{\lambda}\left(\prod_{j=1}^{l} c_{\theta_{j}}\left(x_{j}\right) \prod_{i=1}^{m} c_{\theta_{l}-\frac{\pi}{2}}\left(y_{i}\right) \mid C_{0} \cup\left(\mathbb{R}^{2}-B_{n^{\prime}}\right)\right)(\eta),
$$

for $n^{\prime}$ so large that $x_{j}$ and $y_{i}$ are all contained in $B_{n^{\prime}}$. However (6.18) is obviously a martingale in $n^{\prime}$ and thus (6.18) converges as $n^{\prime} \rightarrow \infty$ for $\mu_{\lambda}$-almost all $\eta$. From (6.5) we also have that (6.17) converges for $\mu_{\lambda}$-almost all $\eta$. Hence we have proved that $\tilde{S}_{C_{0} \cup C_{n^{\prime}}}^{\eta}\left(w,\{\theta\}_{k}\right)$ converges as $n^{\prime} \rightarrow \infty$ for $\mu_{\lambda}$-almost all $\eta$. By Lemma 6.2 we then have that $S_{C_{0} \cup C_{n^{\prime}}}^{\eta}\left(w,\{\theta\}_{h}\right)$ also converges to the same limit. Since $S_{C_{0} \cup C_{n^{\prime}}}^{\eta}\left(w,\{\theta\}_{k}\right)$ is $B_{C_{0}}{ }^{-}$ measurable we get that the common limit is also $B_{C_{0}}$-measurable. By $(6.15),(6.16)$ and (6.18) we have that this common limit is

$$
E_{\lambda}\left(\left[\prod_{j=1}^{k} c_{\theta_{j}}\left(x_{j}\right)\right]\left(\xi-\psi_{\eta}^{C_{0}}\right) \mid B_{C_{0}}^{\infty}\right)(\eta),
$$

where $B_{C_{0}}^{\infty}=\bigcap_{n} B_{C_{0} \cup\left(\mathbb{R}^{2}-B_{n}\right)}$. Hence we have proven that the functions (6.19) are $B_{C_{0}}$-measurable. Making use of (6.16) and of the fact that $\psi_{\eta}^{C_{0}}(x)$ is $B_{C_{0}}$-measurable we get that

$$
E_{\lambda}\left(\left[\prod_{j=1}^{k} c_{\theta_{j}}\left(x_{j}\right)\right](\xi) \mid B_{C_{0}}^{\infty}\right)(\eta)
$$

is $B_{C_{0}}$-measurable. From the fact that the fields $c_{\theta}(x)$ generate the whole $\sigma$-algebra of $\mu_{\lambda}$-measurable sets we get that, for any bounded continuous function $f$ on $S^{\prime}\left(\mathbb{R}^{2}\right)$,

$$
E_{\lambda}\left(f \mid B_{C_{0}}^{\infty}\right)(\eta)
$$

is $B_{C_{0}}$-measurable. Since $B_{C_{0}} \subset B_{C_{0}}^{\infty}$ we may express this in the form

$$
E_{\lambda}\left(f \mid B_{C_{0}}\right)(\eta)=E_{\lambda}\left(f \mid B_{C_{0}}^{\infty}\right)(\eta),
$$

for $\mu_{\lambda}$-almost all $\eta$, because

$$
E_{\lambda}\left(f \mid B_{C_{0}}^{\infty}\right)(\eta)=\lim _{n \rightarrow \infty} E_{\lambda}\left(f \mid C_{0} \cup\left(\mathbb{R}^{2}-B_{n}\right)\right)(\eta),
$$

for $\mu_{\lambda}$-almost all $\eta$. We have thus proven the following Theorem, which is actually a stronger version of the uniqueness Theorem 5.1.

Theorem 6.1 Let $\alpha^{2}<2 \pi$ and $m_{0}>0$, then there exists a number $\lambda_{1}>0$, depending only on $\alpha$ and $m_{0}$, such that, for $-\lambda_{1} \leqq \lambda \leqq \lambda_{1}$ and for any piecewise $C^{1}$-curve $C_{0}$ and any bounded continuous function $f$ on $S^{\prime}\left(\mathbb{R}^{2}\right)$, we have $E_{\lambda}\left(f \mid C_{0}\right)(\eta)$

$=\lim _{n \rightarrow \infty} E_{\lambda}\left(f \mid C_{0} \cup\left(\mathbb{R}^{2}-B_{n}\right)\right)(\eta)$, for $\mu_{\lambda}$-almost all $\eta$, where $B_{n} \equiv\left\{x \in \mathbb{R}^{2}|| x \mid \leqq n\right\}$ and $\mu_{\lambda}$ is the unique regular random field of Theorem 5.1. 
Let now $C_{0}$ be a piecewise $C^{1}$-curve such that $\mathbb{R}^{2}-C_{0}$ consists of two components $\Omega_{+}$and $\Omega_{-}$. Let, for some fixed $n_{0}, f_{+}$and $f_{-}$be bounded continuous functions which are $B_{\Omega_{+}} \cap B_{n_{0}}$ respectively $B_{\Omega_{-}} \cap B_{n_{0}}$-measurable.

By Theorem 6.1 we then have for $\mu_{\lambda}$-almost all $\eta$

$$
E_{\lambda}\left(f_{+} f_{-} \mid C_{0}\right)(\eta)=\lim _{n \rightarrow \infty} E_{\lambda}\left(f_{+} f_{-} \mid C_{0} \cup\left(\mathbb{R}^{2}-B_{n}\right)\right)(\eta) .
$$

By the local Markov property (Theorem 3.1) we have that, for $n \geqq n_{0}$ and $\mu_{\lambda^{-}}$ almost all $\eta$,

$$
\begin{aligned}
& E_{\lambda}\left(f_{+} f_{-} \mid C_{0} \cup\left(\mathbb{R}^{2}-B_{n}\right)\right)(\eta)=E_{\lambda}\left(f_{+} f_{-} \mid C_{0} \cup C_{n}\right)(\eta) \\
& \quad=E_{\lambda}\left(f_{+} \mid C_{0} \cup C_{n}\right)(\eta) E_{\lambda}\left(f_{-} \mid C_{0} \cup C_{n}\right)(\eta) \\
& \quad=E_{\lambda}\left(f_{+} \mid C_{0} \cup\left(\mathbb{R}^{2}-B_{n}\right)\right)(\eta) E_{\lambda}\left(f_{-} \mid C_{0} \cup\left(\mathbb{R}^{2}-B_{n}\right)\right)(\eta)
\end{aligned}
$$

with $C_{n} \equiv \partial B_{n}$. From Theorem 6.1 we then get

$$
E_{\lambda}\left(f_{+} f_{-} \mid C_{0}\right)(\eta)=E_{\lambda}\left(f_{+} \mid C_{0}\right)(\eta) E_{\lambda}\left(f_{-} \mid C_{0}\right)(\eta) .
$$

Hence we have proven the following Theorem, giving the global Markov property for trigonometric interactions:

Theorem 6.2. Let $\alpha^{2}<2 \pi$ and $m_{0}>0$, then there exists a number $\lambda_{1}>0$ depending only on $\alpha$ and $m_{0}$ such that, for $-\lambda_{1} \leqq \lambda \leqq \lambda_{1}$, the measure $\mu_{\lambda}$ given by Theorem 5.1 has the global Markov property. This is to say that for any piecewise $C^{1}$-curve $C_{0}$ such that $\mathbb{R}^{2}-C_{0}$ has two components $\Omega_{+}$and $\Omega_{-}$and for any bounded continuous functions $f_{+}$and $f_{-}$which are $B_{\Omega_{+}}$-respectively $B_{\Omega_{-}}$-measurable one has

$$
E_{\lambda}\left(f_{+} f_{-} \mid C_{0}\right)(\eta)=E_{\lambda}\left(f_{+} \mid C_{0}\right)(\eta) E_{\lambda}\left(f_{-} \mid C_{0}\right)(\eta)
$$

for $\mu_{\lambda}$-almost all $\eta$. We recall that, for any Borel-measurable set $\Lambda \subset \mathbb{R}^{2}, B_{\Lambda}$ denotes the $\sigma$-algebra generated by $\mu_{\lambda}$-null sets and the linear functions $\xi \rightarrow\langle\varrho, \xi\rangle$, where $\varrho$ is any measure of bounded support with supp $\varrho \subset A$ and finite energy, i.e. such that $\int G_{m}(x-y) d \varrho(x) d \varrho(y)<\infty$, for some $m>0$. Moreover $E_{\lambda}\left(f \mid C_{0}\right)$ stands for the conditional expectation $E_{\lambda}\left(f \mid B_{C_{0}}\right)$.

Acknowledgements. We are very grateful to Dr. J. Bellissard and Prof. J. Fröhlich for helpful discussions and corrections, and to Professor E. Dynkin and Professor R. Dobrushin for their steady interest and encouragement concerning the prolems of this paper. We also thank Professor R. Stora and all members of the Centre de Physique Théorique du CNRS, Marseille, for their warm hospitality, as well as the Mathematics Departments of the Universities of Bielefeld and Oslo for kind invitations, during which some of this work was done. We are also grateful to Mrs. Burghardt for her patience and fine typing.

\section{References}

1. Nelson, E.: The free Markov field, J. Funct. Anal. 12, 221-227 (1973)

2. Glimm, J., Jaffe, A., Spencer, E.: The particle structure of the weakly coupled $P(\varphi)_{2}$ model and other applications of high-temperature expansions. In: Constructive quantum field theory. Velo, G., Wightman, A., (eds.) Lecture notes in physics, Vol. 25, pp. 132-242. Berlin, Heidelberg, New York: Springer 1973

3. Albeverio, S., Høegh-Krohn, R.: The Wightman axioms and the mass gap for strong interactions of exponential type in twodimensional space-time. J. Funct. Anal. 16, 39 82 (1974) 
4. Fröhlich, J., Seiler, E.: The massive Thirring-Schwinger model $\left(Q E D_{2}\right)$ : convergence of perturbation theory and particle structure. Helv. Phys. Acta 49, 889-924 (1976)

5.a) Albeverio, S., Høegh-Krohn, R.: Topics in infinite dimensional analysis. In: Mathematical problems in theoretical physics. Dell'Antonio, G.F., Doplicher, S., Jona-Lasinio, G. (eds.) Lecture notes in physics, Vol. 80, pp. 279-302. Berlin, Heidelberg, New York: Springer 1978

b) Albeverio, S., Høegh-Krohn, R.: Local and global Markov fields. Lectures given at Karpacz Winter School, 1978

6.a) Simon, B.: The $P(\phi)_{2}$ Euclidean (quantum) field theory. Princeton: Princeton University Press 1974

b) Klein, A.: The semigroups characterization of Osterwalder-Schrader path spaces and the construction of Euclidean fields. J. Funct. Anal. 27, 277-291 (1978)

7. Nelson, E. : Construction of quantum fields from Markoff fields. J. Funct. Anal. 12, 97-112 (1973)

8. Newman, C.: The construction of stationary twodimensional Markoff fields with an application to quantum field theory. J. Funct. Anal. 12, 211-227 (1973)

9.a) Royer, G., Yor, M.: Représentation intégrale de certaines mesures quasi-invariantes sur $C(R)$; mesures extrémales et propriété de Markov. Ann. Inst. Fourier 26, 7-24 (1976)

b) Dang Ngoc, N., Royer, G. : Markov property of extremal local fields. Proc. Am. Math. Soc. 70, 185-188 (1978)

10. Osterwalder, K., Schrader, R. : Axioms for Euclidean Green's functions II. Commun. Math. Phys. 42, 281-305 (1975)

11.a) Fröhlich, J.: Verification of axioms for Euclidean and relativistic fields and Haag's theorem in a class of $P(\phi)_{2}$-models. Ann. Inst. Henri Poincaré 21, 271-317 (1974)

b) Hegerfeld, G.C.: From Euclidean to relativistic fields and on the notion of Markoff fields. Commun. Math. Phys. 35, 155-171 (1974)

c) Challifour, J.L. : Euclidean field theory. II. Remarks on embedding the relativistic Hilbert space. J. math. Phys. 17, 1889-1892 (1976)

d) Yngvason, J. : On the decomposition of Wightman functionals in the Euclidean framework. Rep. Math. Phys. 13. 101-115 (1978)

12. Accardı, L. : Local perturbations of conditional expectations. Preprint Marseille (1977)

13. Dobrushin, R.L.: Description of a random field by means of conditional probabilities and conditions of its regularity. Theory Probab. Its Appl. USSR 13, 197-224 (1968) (transl.)

14.a) Dobrushin, R.L. : Prescribing a system of random variables by conditional expectation. Theory Probab. Its Appl. USSR 15, 3, 458-486 (1970)

b) Dobrushin, R.L.: Gibbsian random fields for particles without hard core. Theor. Math. Phys. 4. 101-118 (1970) (trans1.)

c) Lanford, O.E.: Entropy and equilibrium states in classical statistical mechanics. In: Statistical mechanics and mathematical problems. Lenard, A. (ed.). Lecture notes in physics, Vol. 20, Berlin, Heidelberg, New York: Springer 1973

d) Dobrushin, R.L.: Conditions for the absence of phase transitions in one-dimensional classical systems. Mat. Sb. 93, 28-48 (1974)

e) Benfatto, G., Presutti, E., Pulvirenti, M.: DLR measures for one-dimensional harmonic systems. Z. Wahrscheinlichkeitstheorie verw. Gebiete 41, 305-312 (1978)

f) Cassandro, M., Olivieri, E., Pellegrinotti, A., Presutti, E.: Existence and uniqueness of DLR measures for unbounded spin systems. Z. Wahrscheinlichkeitstheorie verw. Gebiete 41, 313-334 (1978)

g) Royer, G.: Etude des champs Euclidiens sur un réseau $\mathbb{Z}^{v}$. J. Math. Pures Appl. (to appear)

h) Bellissard. J., Picco, P.: Lattice quantum fields: uniqueness and Markov property. CNRS-CPT Preprint, Dec. 1978

i) See also for related work Preston, C.: Random fields. Lecture notes in mathematics, Vol. 534. Berlin, Heidelberg, New York: Springer 1976 and references therein

15.a) Dobrushin, R.L., Minlos, R. : Construction of a one dimensional quantum field via a continuous Markov field. Funct. Anal. Its Appl. 7, 81-82 (1973); (transl. 7, 324-325 (1973))

15.b) Dobrushin, R.L., Minlos, R.: The theory of Markov random fields and its applications to quantum field theory. In: Functional and probabilistic methods in quantum field theory, Vol. I, pp. 23-49. Jancewicz, B. (ed.). Acta Univ. Wratisl. No. 368, XII-th Winter School of Theor. Phys., Karpacz, 1975; Wroclaw 1976 
16. Guerra, F., Rosen, L., Simon, B. : The $P(\varphi)_{2}$ Euclidean quantum field theory as classical statistical mechanics. Ann. Math. 101, 111-259 (1975)

17. Albeverio, S., Høegh-Krohn, R. : Canonical relativistic quantum fields. Ann. Inst. Henri Poincaré A (to appear)

18.a) Coester, F., Haag, R.: Representation of states in a field theory with canonical variables. Phys. Rev. 117, 1137-1145 (1960)

b) Araki, H.: Hamiltonian formalism and the canonical commutation relations in quantum field theory. J. Math. Phys. 1, 492-504 (1960)

19.a) Albeverio, S., Høegh-Krohn, R.: Dirichlet forms and diffusion processes on rigged Hilbert spaces. Z. Wahrscheinlichkeitstheorie verw. Gebiete 40, 1-57 (1977)

b) Albeverio, S., Høegh-Krohn, R.: Hunt processes and analytic potential theory on rigged Hilbert spaces. Ann. Inst. Henri Poincaré B 13, 269-291 (1977)

20. Lanford III, O.E., Ruelle, D.: Observables at infinity and states with short range correlations in statistical mechanics. Commun. Math. Phys. 13, 194-215 (1969)

21. Albeverio, S., Høegh-Krohn, R.: Uniqueness of the physical vacuum and the Wightman functions in the infinite volume limit for some non-polynomial interactions. Commun. Math. Phys. 30, 171-200 (1973)

22. Fröhlich, J.: Classical and quantum statistical mechanics in one and two dimensions, twocomponent Yukawa- and Coulomb systems. Commun. Math. Phys. 47, 233-268 (1976)

23. Fröhlich, J., Park, Y.M.: Remarks on exponential interactions and the quantum sine-Gordon equation in two space-time dimensions. Helv. Phys. Acta 50, 315-329 (1977)

24. Albeverio, S., Høegh-Krohn, R., Olsen, G.: The global Markov property for lattice systems. Preprint, Bielefeld-Marseille (Nov. 1978)

25. Frohlich, J.: Schwinger functions and their generating functionals, I. Helv. Phys. Acta 74, 265 306 (1974); II. Adv. Math. 23, 119-180 (1977)

26. Guerra, F., Rosen, L., Simon, B. : Boundary conditions for the $P(\varphi)_{2}$ Euclidean field theory. Ann. Inst. Henri Poincaré A 25, 231-334 (1976)

27. Fröhlich, J., Simon, B.: Pure states of general $P(\varphi)_{2}$ theories, construction, regularity and variational equality. Ann. Math. 105, 493-526 (1977)

28. Guerra, F.: External field dependence of magnetization and long range order in quantum field theory. Proc. Bielefeld Symp. Streit, L. (ed.). Wien, New York: Springer 1976

29. Meyer, P.A.: Probabilités et potentiel. Paris: Herman 1966

30. Ciesielski, Z.: Lectures on Brownian motion, heat conduction and potential theory. Lecture notes, No. 3. Aarhus: Mathematics Institute, Aarhus University 1965

Communicated by A. Jaffe

Received October 3, 1978 